\title{
AN UNCOMMON PYRENE TYPE AND TWO NEW GENERA IN THE NEOTROPICAL PSYCHOTRIEAE (RUBIACEAE)
}

\author{
A. L. Borhidi ${ }^{1}$, E. Martínez Salas ${ }^{2}$ and C. H. Ramos Álvarez ${ }^{3}$ \\ ${ }^{1}$ Department of Plant Biology, Institute of Biology, Faculty of Sciences \\ University of Pécs, Pécs, Hungary; E-mail: borhidi@gamma.ttk.pte.hu \\ ${ }^{2}$ Herbario Nacional de México, Instituto de Biología \\ Universidad Nacional Autónoma de México (UNAM), México; E-mail: ems@ib.unam.mx \\ ${ }^{3}$ Av. IMAN 580, Helios 101, México, D. F., C. P. 4510, México \\ E-mail: clara.hilda.ramos@gmail.com
}

(Received 29 April, 2015; Accepted 10 July, 2015)

\begin{abstract}
A new pyrene type is reported, it is morphologically different from that found in the genera Palicourea and Psychotria. This new type is characterised by being triangulate in transverse section with an elevated central-dorsal crest. It was observed in two species of Palicourea with dissimilar floral characteristics. Based on this finding, we propose to transfer Palicourea tetragona (Donn.-Sm.) C. M. Taylor et Lorence to Mexocarpus gen. nov., and Palicourea seemannii Standl. to Ditrichanthus gen. nov.
\end{abstract}

Resumen. Se ha detectado un nuevo tipo de pireno morfológicamente distinto de los ampliamente conocidos en los géneros Palicourea y Psychotria. El nuevo tipo se caracteriza por presentar sección transversal triangular y una cresta dorsal-central muy prominente; contrastando con la sección transversal semicircular, dorsalmente lisa o inconspicuamente 3-5 costulada de los géneros ya mencionados. Este tipo de pireno se observó en dos especies de Palicourea con características florales diferentes entre sí. Con base en estos hallazgos se proponen dos géneros nuevos: Mexocarpus basado en Palicourea tetragona (Donn.-Sm.) C. M. Taylor et Lorence y Ditrichanthus apoyado en Palicourea seemannii Standl.

Key words: Ditrichanthus, fruit morphology, Latin America, Mexocarpus, Rubiaceae

\section{INTRODUCTION}

Robbrecht emphasised in several papers the high diagnostic value of pyrene characters in the Rubiaceae particularly in the tribe Psychotrieae (Robbrecht 1975, 1988, 1989a, b). He referred to prior studies of the basic importance of fruit characteristics, which turned out to be very useful in the classification of the African Psychotrieae. Petit (1964) observed a wide array of morphological variation in fruit and seeds; the pyrenes proved to be extremely variable in size, shape, occurrence of appendages, etc., and the performed germina- 
tion slits (PGSs) on the ventral surface of the pyrenes. He found good correlations between the variation of PGSs (number, position, length, etc.) and other characters. He concluded that PGS characters have a high diagnostic value, particularly at the generic level, and summarised his observations in a key to the African genera of Psychotrieae and related tribes. According to this key (Petit 1963, Robbrecht 1989a), African Psychotria species are characterised by absence of germination slits; in contrast, neotropical Palicourea and Psychotria s. 1. species do have a ventral longitudinal germination slit (Taylor 2012a, b).

\section{THE NEW PYRENE TYPE}

Morphological characters of fruit and pyrenes are rather uniform among the approximately 200 species of the genus Palicourea and 800 neotropical species of Psychotria. Fruits are drupaceous, globose, subglobose, elliptical or ovoid-oblong, with fleshy to juicy mesocarp; each usually contains 2 pyrenes (5 in a few species), 1-locular, plane convex, always hemispherical with semiorbicular cross section (obtriangular when 5), generally with 3-5 obtuse or acute longitudinal ribs on the dorsal surface, and a longitudinal central ventral slit on the inner plane, or slightly concave surface. However, these combination of characters have been studied and verified by de first author in fruits of 323 neotropical Palicourea and Psychotria species (Table 1). During the process with careful observation a particularly different pyrene type was found in two species: Palicourea tetragona (Donn.-Sm.) C. M. Taylor et Lorence and P. seemannii Standl. One of them Palicourea tetragona was originally described in the genus Cephaëlis, later transferred to Psychotria and recently to Palicourea. The fruits are drupaceous, globose, subglobose or obovate, slightly to explic-

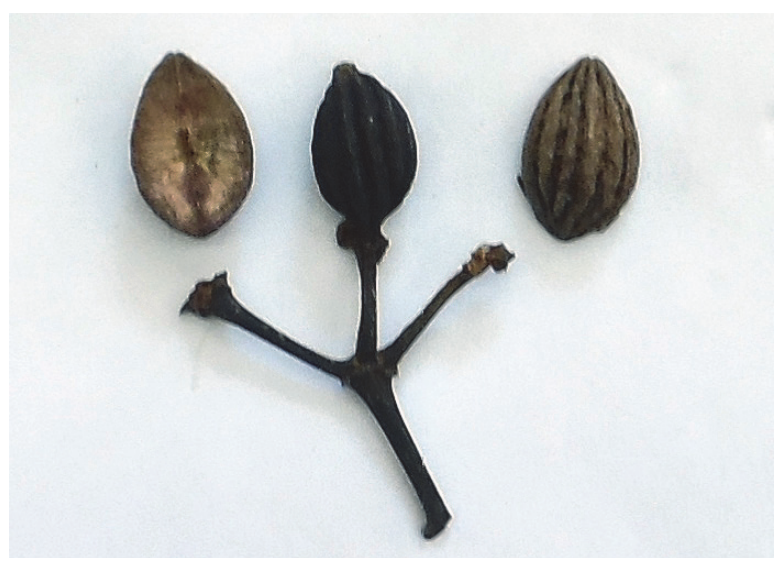

Fig. 1. The common form of pyrenes in the Palicourea and Psychotria genera. Example: pyrenes of Palicourea calophylla (Stevens et al. 25581, MEXU) (photo: A. Borhidi) 
Table 1

Morphological characters of fruit and pyrenes in neotropical Palicourea and Psychotria species

\begin{tabular}{|c|c|c|c|c|}
\hline Species & $\begin{array}{l}\text { Drupe size } \\
\quad(\mathrm{mm})\end{array}$ & Shape & $\begin{array}{c}\text { Pyrene } \\
\text { dorsal ribs }\end{array}$ & $\begin{array}{l}\text { Sec- } \\
\text { tion }\end{array}$ \\
\hline \multicolumn{5}{|c|}{ Mesoamerican Palicourea species } \\
\hline P. acetosoides Wernh. & $\begin{array}{c}3.5-4.5 \times \\
3.5-4.5\end{array}$ & elliptical-subglobose & 4-5 rounded & hsf \\
\hline P. adusta Standl. & $\begin{array}{c}3.5-4.5 \times \\
3.5-4.5\end{array}$ & elliptical-obovate & 4-5 rounded & hsf \\
\hline P. alajuelensis C. M. Taylor & $10-12$ & subglobose & $\begin{array}{l}\text { smooth or } \\
3-4 \text { weak }\end{array}$ & hsf \\
\hline P. albocaerulea C. M. Taylor & $5-6 \times 5-6$ & ovoidal & 3-5 rounded & hsf \\
\hline P. angustifolia Kunth in HBK. & $4-6 \times 4-6$ & & 3-5 rounded & hsf \\
\hline P. beachiana C. M. Taylor & $9 \times 10$ & elliptical & $3-4$ acute & hsf \\
\hline P. bella (Standl.) Dwyer & $6 \times 6$ & elliptical & $3-4$ acute & hsf \\
\hline P. bellula C. M. Taylor & $5 \times 5$ & subglobose & $3-5$ acute & hsf \\
\hline P. breedlovei (Lorence) Lorence & 6-8 (diam.) & & $\begin{array}{c}\text { 3-5 obtuse, } \\
\text { weak }\end{array}$ & hsf \\
\hline P. brenesii Standl. & $4-6 \times 4-6$ & subglobose & 3-5 rounded & hsf \\
\hline P. calidicola (Taylor) Taylor & $15-20 \times 12-20$ & obovate-elliptical & $\begin{array}{c}\text { 4-5 obtuse, } \\
\text { weak }\end{array}$ & hsf \\
\hline P. chiriquina Standl. & $3.5-5 \times 3.5-4$ & obovate & $3-5$ rounded & hsf \\
\hline P. chrysocalymma (L. O. Wms.) Taylor & 5 (diam.) & & $\begin{array}{c}\text { 3-4 obtuse, } \\
\text { weak }\end{array}$ & hsf \\
\hline P. crocea (Sw.) Roem. et Schult. & $4-6 \times 4-6$ & obovate-globose & $3-5$ rounded & hsf \\
\hline P. dimorphandroides (Dwyer) Taylor & $5 \times 4$ & elliptical-ovate & $3-5$ acute & hsf \\
\hline P. discolor K. Krause & $4-5 \times 4-5$ & subglobose & $3-5$ rounded & hsf \\
\hline P. domingensis (Jacq.) DC. & $4-7 \times 6-8$ & oval-elliptical & $4-5$ acute & hsf \\
\hline P. eurycarpa (Standl.) Taylor & 8-12 (diam.) & elliptical-obovate & $4-5$ obtuse & hsf \\
\hline P. garciae Standl. & $5 \times 5$ & elliptical & $3-5$ rounded & hsf \\
\hline P. gardenioides (Scheidw.) Hemsl. & $5.5-6 \times 6$ & elliptical & $4-5$ acute & hsf \\
\hline P. gomezii C. M. Taylor & $6 \times 6$ & obovate & $3-5$ acute & hsf \\
\hline P. grandifructa (Taylor) Taylor & 15 (diam.) & elliptical & $\begin{array}{l}\text { smooth or } \\
4-5 \text { weak }\end{array}$ & hsf \\
\hline P. guianensis Aubl. & $5-7 \times 3.5-5$ & ovate-elliptical & $3-5$ rounded & hsf \\
\hline P. hammelii C. M. Taylor & $8-10 \times 7-8$ & elliptical & $3-4$ acute & hsf \\
\hline P. heydei (Standl.) Lorence & $7 \times 6$ & elliptical & $4-5$ acute & hsf \\
\hline P. hondensis (Standl.) Taylor & 15-17 (diam.) & subglobose-elliptical & $\begin{array}{l}\text { smooth to } \\
\text { angulate }\end{array}$ & hsf \\
\hline P. ianthina C. M. Taylor & $7 \times 4$ & obovate-elliptical & $3-5$ rounded & hsf \\
\hline
\end{tabular}


Table 1 (continued)

\begin{tabular}{|c|c|c|c|c|}
\hline Species & $\begin{array}{l}\text { Drupe size } \\
(\mathrm{mm})\end{array}$ & Shape & $\begin{array}{l}\text { Pyrene } \\
\text { dorsal ribs }\end{array}$ & $\begin{array}{l}\text { Sec- } \\
\text { tion }\end{array}$ \\
\hline P. lancifera Standl. et L. O. Wms. & $4-5 \times 4-5$ & ovate-subglobose & $4-5$ rounded & hsf \\
\hline P. lasiorrhachis Oerst. & $4-5 \times 3-5$ & obovate & $4-5$ rounded & hsf \\
\hline P. leucantha Donn.-Sm. & $4-6 \times 3-5$ & elliptical & $4-5$ rounded & hsf \\
\hline P. macrantha Loes. & $5 \times 4-4.5$ & elliptical-ovate & $3-5$ rounded & hsf \\
\hline P. macrocalyx Standl. & $5-6 \times 4-5$ & elliptical-subglobose & $4-5$ rounded & hsf \\
\hline $\begin{array}{l}\text { P. mediocris (Standl. et Steyerm.) } \\
\text { Lorence }\end{array}$ & 10 (diam.) & subglobose & smooth & hsf \\
\hline P. montivaga Standl. & $4-5 \times 3-4$ & elliptical-obovate & $4-5$ rounded & hsf \\
\hline P. nebulosa (Dwyer) Taylor & $10-12 \times 10-12$ & elliptical & $4-5$ acute & hsf \\
\hline P. neopurpusii C. M. Taylor & $7 \times 6$ & elliptical-ovate & $4-5$ acute & hsf \\
\hline P. ochnoides Dwyer & $5-7 \times 5-7$ & elliptical-subglobose & $4-5$ acute & hsf \\
\hline P. orosiana C. M. Taylor & $5 \times 5$ & obovate & $4-5$ rounded & hsf \\
\hline $\begin{array}{l}\text { P. padifolia (Willd. ex Roem. et } \\
\text { Schult.) C. M. Taylor et Lorence }\end{array}$ & $\begin{array}{l}4-6(10) \times \\
3-6(10)\end{array}$ & ovate-elliptical & $4-5$ rounded & hsf \\
\hline P. palustris A. C. Gilman et Taylor & $5 \times 5$ & elliptical & $\begin{array}{l}3-4 \text { broadly } \\
\text { angulate }\end{array}$ & hsf \\
\hline P. pauciflora Standl. & $7 \times 6-7$ & elliptical-ovate & $4-5$ acute & hsf \\
\hline P. pendula C. M. Taylor & $6-7 \times 4-4.5$ & obovate & $4-5$ rounded & hsf \\
\hline P. pereziana C. M. Taylor & $8-10 \times 8-10$ & elliptical-obovate & $\begin{array}{l}3-5 \text { broadly } \\
\text { angulate }\end{array}$ & hsf \\
\hline $\begin{array}{l}\text { P. providenciana Sanchez-Gonz. et } \\
\text { Taylor }\end{array}$ & $4-6 \times 4-5$ & obovate-elliptical & $3-4$ rounded & hsf \\
\hline $\begin{array}{l}\text { P. psychotrioides (Taylor et Hammel) } \\
\text { Taylor }\end{array}$ & $15-20 \times 12-16$ & elliptical & $\begin{array}{l}\text { smooth or } \\
4-5 \text { weak }\end{array}$ & hsf \\
\hline P. purpurea C. M. Taylor & $4-5 \times 5-6$ & subglobose & $4-5$ rounded & hsf \\
\hline P. pyramidalis Standl. & $5-6 \times 5-6$ & obovate & $4-5$ rounded & hsf \\
\hline $\begin{array}{l}\text { P. rigidifolia (Dwyer et Hayden) } \\
\text { Dwyer }\end{array}$ & $5-7 \times 5-10$ & subglobose-elliptical & smooth & hsf \\
\hline P. roseocrema (Dwyer) C. M. Taylor & $9 \times 10$ & elliptical & $4-5$ acute & hsf \\
\hline P. roseofaucis C. M. Taylor & $5 \times 5.5$ & elliptical-obovate & $\begin{array}{l}3-4 \text { broadly } \\
\text { angulate }\end{array}$ & hsf \\
\hline P. salicifolia Standl. & $6-7 \times 6-7$ & elliptical & $\begin{array}{l}\text { 3-4 broadly } \\
\text { angulate }\end{array}$ & hsf \\
\hline P. skotaki C. M. Taylor & $6-7 \times 5$ & elliptical-obovate & $4-5$ acute & hsf \\
\hline P. spathacea C. M. Taylor & $6-8 \times 6-8$ & elliptical-subglobose & $3-5$ acute & hsf \\
\hline P. standleyana C. M. Taylor & $8 \times 5$ & elliptical & $3-5$ rounded & hsf \\
\hline P. thornei (Lorence) Lorence & $7-8 \times 4-5$ & elliptical & 4 rounded & hsf \\
\hline
\end{tabular}


Table 1 (continued)

\begin{tabular}{|c|c|c|c|c|}
\hline Species & $\begin{array}{l}\text { Drupe size } \\
(\mathrm{mm})\end{array}$ & Shape & $\begin{array}{l}\text { Pyrene } \\
\text { dorsal ribs }\end{array}$ & $\begin{array}{l}\text { Sec- } \\
\text { tion }\end{array}$ \\
\hline P. tilaranensis C. M. Taylor & $4-5 \times 3.4$ & elliptical & $4-5$ rounded & hsf \\
\hline P. triphylla DC. & $3.5-5 \times 3.5-4$ & ovate & $3-5$ rounded & hsf \\
\hline P. tubuliflora Dwyer & $8-10 \times 6-8$ & elliptical-subglobose & smooth & hsf \\
\hline $\begin{array}{l}\text { P. umbelliformis (Dwyer et Hayden) } \\
\text { Taylor }\end{array}$ & $15 \times 12$ & elliptical & $4-5$ acute & hsf \\
\hline P. vestita Standl. & $6 \times 6$ & obovate & $4-5$ rounded & hsf \\
\hline \multicolumn{5}{|c|}{ Mexican and Mesoamerican Heteropsychotria species } \\
\hline H. acicularis C. M. Taylor & 4 (diam.) & elliptical-didymous & $3-5$ obtuse & hsf \\
\hline H. acuminata Benth. & $3-5 \times 5-6$ & subglobose & smooth & hsf \\
\hline H. allenii Standl. & 14-15 (diam.) & elliptical & smooth & hsf \\
\hline H. angustiflora K. Krause & 5-7 (diam.) & elliptical-ovate & $4-5$ acute & hsf \\
\hline H. aubletiana Steyerm. & $3-8 \times 2-5$ & elliptical-ovate & 3-4 obtuse & hsf \\
\hline H. aurantibractea C. M. Taylor & $5 \times 4.5$ & subglobose & $3-4$ acute & hsf \\
\hline H. berteroana DC. & $3.5-5$ & elliptical-didymous & $4-5$ weak & hsf \\
\hline H. boraginoides (Dwyer) Taylor & $5 \times 7$ & elliptical-obovate & smooth & hsf \\
\hline H. brachiata Sw. & $4-5 \times 3.5$ & subglobose & $3-5$ acute & hsf \\
\hline H. buchtienii (H. J. P. Winkl.) Standl. & $4.5-5$ (diam.) & elliptical-subglobose & $4-5$ acute & hsf \\
\hline H. caerulea Ruiz et Pav. & $5 \times 4$ & subglobose-elliptical & $4-5$ obtuse & hsf \\
\hline H. capitata Ruiz et Pav. & 5-7 (diam.) & elliptical & $3-5$ acute & hsf \\
\hline H. carnosocarpa Dwyer et Hayden & $4-5 \times 5.5-6$ & elliptical-obovate & smooth & hsf \\
\hline H. chiriquiensis (Standl.) Taylor & $5 \times 4$ & elliptical & smooth & hsf \\
\hline H. chlorobotrya Standl. & $4-5$ (diam.) & obovate & $4-5$ acute & hsf \\
\hline H. cooperi Standl. & $5 \times 3.5$ & elliptical-obovate & $3-5$ acute & hsf \\
\hline H. correae (Dwyer et Hayden) Taylor & $10-15 \times 5-6$ & elliptical & 3-4 weak & hsf \\
\hline H. croatii (Dwyer) Taylor & $4 \times 3.5$ & subglobose & $4-5$ obtuse & hsf \\
\hline H. croceovenosa Dwyer & 3.5-4 (diam.) & elliptical & $4-5$ acute & hsf \\
\hline H. cyanococca Seem. et Dombrain & $3-4 \times 2.5-3.5$ & subglobose-elliptical & $4-5$ rounded & hsf \\
\hline H. deflexa DC. & $\begin{array}{l}2.5-3.5 \\
\text { (diam.) }\end{array}$ & obovate & $4-5$ obtuse & hsf \\
\hline H. dichroa (Standl.) C. M. Taylor & $8 \times 5.5$ & elliptical & $3-4$ rounded & hsf \\
\hline H. elata (Sw.) Hammel & $5-10 \times 2-5$ & elliptical & $3-4$ rounded & apl \\
\hline H. gaitalensis C. M. Taylor & $3 \times 2.5$ & elliptical & $4-5$ acute & hsf \\
\hline $\begin{array}{l}\text { H. galeottiana (M. Martens) Taylor } \\
\text { et Lorence }\end{array}$ & $3-4 \times 3-4$ & elliptical & $4-5$ rounded & hsf \\
\hline H. glomerulata (Donn.-Sm.) Steyerm. & & elliptical & smooth & hsf \\
\hline
\end{tabular}


Table 1 (continued)

\begin{tabular}{|c|c|c|c|c|}
\hline Species & $\begin{array}{l}\text { Drupe size } \\
\quad(\mathrm{mm})\end{array}$ & Shape & $\begin{array}{l}\text { Pyrene } \\
\text { dorsal ribs }\end{array}$ & $\begin{array}{l}\text { Sec- } \\
\text { tion }\end{array}$ \\
\hline H. goldmanii Standl. & $3.5-4 \times 3-3.5$ & elliptical & $3-4$ obtuse & hsf \\
\hline H. gracilenta Müll.-Arg. & $3-4 \times 3-4$ & oblanceolate & 3-4 obtuse & hsf \\
\hline H. guapilensis (Standl.) Hammel & $8-10 \times 6-8$ & elliptical & smooth & hsf \\
\hline H. hazenii Standl. & $4 \times 3.5-4$ & elliptical & 3-4 obtuse & hsf \\
\hline H. hebeclada DC. & $3-5 \times 3-5$ & elliptical-subglobose & $3-5$ obtuse & hsf \\
\hline H. hispidula Standl. et Steyerm. & $4.5-5 \times 4.5$ & elliptical-subglobose & $4-5$ rounded & hsf \\
\hline $\begin{array}{l}\text { H. hoffmannseggiana (Willd. ex } \\
\text { Roem. et Schult.) Müll.-Arg. }\end{array}$ & 4.5 (diam.) & elliptical & $4-5$ rounded & hsf \\
\hline H. juarezana C. M. Taylor et Lorence & $5 \times 4.5$ & subglobose & $4-5$ rounded & hsf \\
\hline H. longicuspis Müll.-Arg. & 4.5-5 (diam.) & subglobose & smooth & hsf \\
\hline H. longirostris (Rusby) Standl. & 4-5 (diam.) & elliptical-obovate & $3-4$ acute & hsf \\
\hline $\begin{array}{l}\text { H. lozadae Borhidi et Lorea-Her- } \\
\text { nandez }\end{array}$ & $4-5$ (diam.) & elliptical-obovate & $3-5$ acute & hsf \\
\hline H. luxurians Rusby & $2.5-3$ (diam.) & subglobose & $4-5$ obtuse & hsf \\
\hline H. microbotrys Ruiz ex Standl. & 3-4.5 (diam.) & elliptical & 3-4 obtuse & hsf \\
\hline H. minarum Standl. et Steyerm. & $4.5-5 \times 4.5$ & ovate & $4-5$ rounded & hsf \\
\hline H. molinarum Lorence & $5 \times 5$ & elliptical & $3-4$ rounded & hsf \\
\hline H. mortoniana Standl. & $2.5-3$ (diam.) & elliptical-ovate & $4-5$ acute & hsf \\
\hline H. muscosa (Jacq.) Steyerm. & $5 \times 4.5$ & elliptical & $3-4$ rounded & hsf \\
\hline H. oreodoxa L. O. Wms. & 2.5 (diam.) & elliptical & $3-4$ obtuse & hsf \\
\hline H. osaensis C. M. Taylor & $2 \times 3$ & subglobose & $4-5$ obtuse & hsf \\
\hline H. paniculata (Aubl.) Raeusch. & 4 (diam.) & obovate & $4-5$ obtuse & scr \\
\hline H. paradichroa C. M. Taylor & $7 \times 4$ & ovate-elliptical & $3-4$ rounded & hsf \\
\hline H. perotensis Cast.-Campos & 4-7 (diam.) & $\begin{array}{l}\text { subglobose- } \\
\text { didymous }\end{array}$ & $3-5$ obtuse & \\
\hline $\begin{array}{l}\text { H. phanaerandra (Standl. et } \\
\text { Steyerm.) Lorence }\end{array}$ & $5-7 \times 5-6.5$ & elliptical & $3-5$ rounded & hsf \\
\hline H. pilosa Ruiz et Pav. & $3-8 \times 3-6$ & elliptical & $3-4$ acute & hsf \\
\hline H. platypoda DC. & $5 \times 5$ & elliptical-cylindrous & smooth & hsf \\
\hline H. poeppigiana Müll.-Arg. & $9-15 \times 3-10$ & elliptical & $3-5$ rounded & hsf \\
\hline H. pubescens Sw. & $3-5 \times 3-5$ & subglobose & $4-5$ obtuse & hsf \\
\hline H. racemosa Rich. & 4-7 (diam.) & & 3 rounded & 3-ang \\
\hline H. recordiana Standl. & 4-4.5 (diam.) & elliptical & $\begin{array}{l}\text { 3-4 obtuse, } \\
\text { weak }\end{array}$ & hsf \\
\hline H. sanblasensis C. M. Taylor & $4 \times 5$ & elliptical & 3-4 obtuse & hsf \\
\hline
\end{tabular}


Table 1 (continued)

\begin{tabular}{|c|c|c|c|c|}
\hline Species & $\begin{array}{l}\text { Drupe size } \\
(\mathrm{mm})\end{array}$ & Shape & $\begin{array}{l}\text { Pyrene } \\
\text { dorsal ribs }\end{array}$ & $\begin{array}{l}\text { Sec- } \\
\text { tion }\end{array}$ \\
\hline H. sanctae-rosae Standl. & $5 \times 4$ & subglobose & 3-4 weak & hsf \\
\hline H. schunkei C. M. Taylor & 7 (diam.) & elliptical-ovate & $3-4$ rounded & hsf \\
\hline H. siccorubra Dwyer & $4-5 \times 4-5$ & $\begin{array}{l}\text { ovate-subglobose- } \\
\text { elliptical }\end{array}$ & $3-4$ rounded & hsf \\
\hline H. simiarum Standl. & $6-8 \times 6-7$ & subglobose-obovate & smooth & hsf \\
\hline H. solitudinum Standl. & 5 (diam.) & elliptical & $\begin{array}{l}4-5 \text { acute to } \\
\text { obtuse }\end{array}$ & hsf \\
\hline H. steyermarkii Standl. & $5 \times 4.5-5$ & obovate & $3-5$ obtuse & hsf \\
\hline H. suerrensis Donn.-Sm. & $4-5$ (diam.) & ovate & $3-5$ obtuse & hsf \\
\hline H. tacarcunensis Dwyer & $4 \times 5-5.5$ & subglobose & $4-5$ obtuse & hsf \\
\hline H. tapantiensis C. M. Taylor & 3.5-4 (diam.) & elliptical-obovate & $4-5$ acute & hsf \\
\hline H. torresiana Standl. & 3-4 (diam.) & subglobose-obovate & $4-5$ acute & hsf \\
\hline H. tsakiana C. M. Taylor & 5 (diam.) & subglobose-oblate & 2-3 rounded & 3-ang \\
\hline H. valerioana Standl. & $5 \times 7$ & subglobose & smooth & hsf \\
\hline H. veracruzensis Lorence et Dwyer & $4-6$ (diam.) & subglobose & 3-4 weak & hsf \\
\hline \multicolumn{5}{|c|}{ Mexican and Mesoamerican Psychotria species } \\
\hline P. aguilarii Standl. et Steyerm. & $5-6 \times 3-4$ & ellipsoidal & smooth & hsf \\
\hline P. alfaroana Standl. & $8-12 \times 4-6$ & ellipsoidal-obovate & $\begin{array}{l}4-5 \text { acute to } \\
\text { rounded }\end{array}$ & hsf \\
\hline P. bakeri Dwyer & $4 \times 4$ & subglobose & $4-5$ rounded & hsf \\
\hline P. calophylla Standl. & $10-14 \times 6-9$ & ellipsoidal & $4-5$ rounded & hsf \\
\hline P. chagrensis Standl. & $5-8 \times 3-6$ & ellipsoid-ovoidal & $4-5$ rounded & hsf \\
\hline $\begin{array}{l}\text { P. chamelaensis C. M. Taylor et } \\
\text { Doming.-Licona }\end{array}$ & $4-5 \times 4-4.5$ & ellipsoidal & $4-5$ obtuse & hsf \\
\hline P. chiriquiana Standl. & $5-6 \times 4-4.5$ & ellipsoidal & $4-5$ acute & hsf \\
\hline P. chitariana Dwyer et C. W. Hamilt. & $6-7 \times 4$ & ellipsoidal & $4-5$ acute & hsf \\
\hline P. clivorum Standl. et Steyerm. & $5-6 \times 4-4.5$ & ellipsoidal & $4-5$ acute & hsf \\
\hline P. cocosensis C. W. Hamilt. & $6 \times 5.5-6$ & $\begin{array}{l}\text { subglobose-ellip- } \\
\text { soidal }\end{array}$ & 4 rounded & hsf \\
\hline P. convergens C. M. Taylor & $6-7 \times 5-6$ & ellipsoidal & $4-5$ rounded & hsf \\
\hline P. costivenia Griseb. & $5-8.5 \times 4-7$ & $\begin{array}{l}\text { ellipsoidal-subglo- } \\
\text { bose }\end{array}$ & $4-5$ rounded & hsf \\
\hline P. dressleri (Dwyer) C. W. Hamilt. & $8-9 \times 3.5-4.5$ & ellipsoidal & $3-5$ rounded & hsf \\
\hline P. durilancifolia Dwyer & $7-8 \times 7$ & subglobose & $4-5$ rounded & hsf \\
\hline P. dwyeri C. M. Hamilt. & $4.5-5$ (diam.) & globose & $4-5$ acute & hsf \\
\hline
\end{tabular}


Table 1 (continued)

\begin{tabular}{|c|c|c|c|c|}
\hline Species & $\begin{array}{l}\text { Drupe size } \\
(\mathrm{mm})\end{array}$ & Shape & $\begin{array}{l}\text { Pyrene } \\
\text { dorsal ribs }\end{array}$ & $\begin{array}{l}\text { Sec- } \\
\text { tion }\end{array}$ \\
\hline P. erythrocarpa Schltdl. & $4.5-6 \times 3-4.5$ & $\begin{array}{l}\text { ellipsoidal-subglo- } \\
\text { bose }\end{array}$ & $3-5$ rounded & hsf \\
\hline P. fendleri Standl. & $4-4.5 \times 3-4$ & $\begin{array}{l}\text { ellipsoidal-subglo- } \\
\text { bose }\end{array}$ & $4-5$ rounded & hsf \\
\hline P. flava Oerst. ex Standl. & $8-13 \times 6-9$ & ellipsoidal & $4-5$ rounded & hsf \\
\hline P. fosteri C. W. Hamilt. & $5-7 \times 2.5-4$ & $\begin{array}{l}\text { ellipsoidal-sublan- } \\
\text { ceolate }\end{array}$ & 5 rounded & hsf \\
\hline P. fruticetorum Standl. & $4-5 \times 3-3.5$ & ellipsoidal & $3-5$ rounded & hsf \\
\hline P. graciliflora Benth. in Oerst. & $4-6 \times 3-5$ & $\begin{array}{l}\text { ellipsoidal-subglo- } \\
\text { bose }\end{array}$ & $3-5$ rounded & hsf \\
\hline P. hamiltoniana C. M. Taylor & $5 \times 3.5$ & ellipsoidal & $3-5$ rounded & hsf \\
\hline P. hammelii Dwyer & $5-7 \times 3.5-4$ & ellipsoidal & $4-5$ acute & hsf \\
\hline P. hidalgensis Borhidi & $5-6 \times 4.5-5$ & elliptical-subglobose & $3-5$ rounded & hsf \\
\hline P. horizontalis Sw. & $3.5-5 \times 2.5-5$ & ellipsoidal & $3-5$ rounded & hsf \\
\hline $\begin{array}{l}\text { P. insueta (Dwyer) Dwyer et C. W. } \\
\text { Hamilt. }\end{array}$ & $7-10 \times 4.5$ & ellipsoidal & ca 5 rounded & hsf \\
\hline P. jefensis Dwyer ex C. M. Taylor & $4 \times 4-4.5$ & subglobose & $4-5$ rounded & hsf \\
\hline P. jimenezii Standl. & $\begin{array}{l}3.5-6 \times \\
3.5-4.5\end{array}$ & elliptical-obovate & $3-4$ rounded & hsf \\
\hline $\begin{array}{l}\text { P. jinotegensis C. Nelson, Molina et } \\
\text { Standl. }\end{array}$ & $5-6.5 \times 4-5$ & ellipsoidal & $4-5$ rounded & hsf \\
\hline P. lamariensis C. W. Hamilt. & $7-8 \times 4-5$ & ellipsoidal & $4-5$ rounded & hsf \\
\hline P. laselvensis C. W. Hamilt. & $4-5 \times 4-5$ & subglobose & $3-5$ rounded & hsf \\
\hline P. liesneri Dwyer & $4-5 \times 4-5$ & subglobose & $4-6$ rounded & hsf \\
\hline P. limonensis K. Krause & $3.5-4.5 \times 3-4$ & ellipsoidal & $4-5$ rounded & hsf \\
\hline P. lorenciana C. M. Taylor & 4-5 (diam.) & subglobose & $4-5$ obtuse & hsf \\
\hline P. lundellii Standl. & $4-5.5 \times 4-5.5$ & subglobose & $\begin{array}{l}\text { smooth to } \\
3-5 \text { plane }\end{array}$ & hsf \\
\hline P. matagalpensis C. M. Taylor & $6-7 \times 4.5-5$ & ellipsoidal-obovoid & $4-5$ rounded & hsf \\
\hline P. mexiae Standl. & $4.5-6 \times 3.5-5$ & subglobose & $4-5$ rounded & hsf \\
\hline P. mirandae C. W. Hamilt. & $5-7 \times 4-5$ & ellipsoidal & 5 rounded & hsf \\
\hline P. molinae Standl. & $5 \times 5-6$ & ellipsoidal & 5 obtuse & hsf \\
\hline P. monsalvae C. M. Taylor & 3.5-4 (diam.) & subglobose & 5 obtuse & hsf \\
\hline $\begin{array}{l}\text { P. monteverdensis Dwyer et C. W. } \\
\text { Hamilt. }\end{array}$ & $8-11 \times 6-12$ & ellipsoidal & $4-5$ rounded & hsf \\
\hline P. neilii C. W. Hamilt. et Dwyer & $5-7 \times 3-3.5$ & ellipsoidal-obovoid & $3-4$ rounded & hsf \\
\hline P. nubiphila Dwyer & $7-8 \times 6-7$ & ellipsoidal & $4-5$ rounded & hsf \\
\hline
\end{tabular}


Table 1 (continued)

\begin{tabular}{|c|c|c|c|c|}
\hline Species & $\begin{array}{l}\text { Drupe size } \\
(\mathrm{mm})\end{array}$ & Shape & $\begin{array}{l}\text { Pyrene } \\
\text { dorsal ribs }\end{array}$ & $\begin{array}{l}\text { Sec- } \\
\text { tion }\end{array}$ \\
\hline $\begin{array}{l}\text { P. oaxacensis Borhidi et Salas- } \\
\text { Morales }\end{array}$ & 5 (diam.) & globose & 4 acute & hsf \\
\hline P. olgae Dwyer et Hayden & $9-12 \times 5.5-8$ & ellipsoidal-obovoid & $3-4$ rounded & hsf \\
\hline P. orosiana Standl. & $4.5-6 \times 3.5-5$ & ellipsoidal & $4-5$ rounded & hsf \\
\hline P. orosioides C. M. Taylor & $4-5 \times 4-5$ & $\begin{array}{l}\text { ellipsoidal-subglo- } \\
\text { bose }\end{array}$ & $4-5$ rounded & hsf \\
\hline P. pacorensis C. W. Hamilt. & $5.5-7 \times 3.5-5$ & ellipsoidal & $4-5$ rounded & hsf \\
\hline P. panamensis Standl. & $4.5-8 \times 3.5-7$ & ellipsoidal-obovoid & $4-5$ rounded & hsf \\
\hline P. papantlensis (Oerst.) Standl. & $6-7.5 \times 4-5$ & ellipsoidal & 4 rounded & hsf \\
\hline P. parvifolia Benth. in Oerst. & $4-5 \times 4-5$ & subglobose & $3-5$ rounded & hsf \\
\hline P. philacra Dwyer & $4.5-5.5 \times 5-6$ & subglobose & $4-5$ acute & hsf \\
\hline P. pisonioides Standl. & $5-7 \times 5-7$ & subglobose & $3-5$ rounded & hsf \\
\hline P. pleuropoda Donn.-Sm. & $5.5-6 \times 4.5-5$ & ellipsoidal & $4-5$ rounded & hsf \\
\hline P. quinqueradiata Pol. & $5.5-9 \times 4-6$ & ellipsoidal & $4-5$ rounded & hsf \\
\hline P. remota Benth. & $6-9 \times 4-6$ & ellipsoidal & $3-4$ rounded & hsf \\
\hline P. rosulatifolia Dwyer & $5-6 \times 4-4.5$ & ellipsoidal & 5 rounded & hsf \\
\hline P. saltatrix C. M. Taylor & $6-6.5 \times 4-4.5$ & obovoidal & $4-5$ acute & hsf \\
\hline P. sarapiquiensis Standl. & $7-10 \times 4-7$ & ellipsoidal-obovoid & $4-5$ acute & hsf \\
\hline P. sixaolensis C. W. Hamilt. & $7-9 \times 5$ & ellipsoidal & 4 acute & hsf \\
\hline P. tenuifolia Sw. & $4-5 \times 3-5$ & subglobose & $4-5$ rounded & hsf \\
\hline P. turrubarensis Burger et $\mathrm{Q}$. Jiménez & $7-10 \times 6-9$ & ovoidal & $4-5$ rounded & hsf \\
\hline P. viridis Ruiz et Pav. & $4-6 \times 3-6$ & $\begin{array}{l}\text { subglobose-ellip- } \\
\text { soidal }\end{array}$ & $4-5$ rounded & hsf \\
\hline \multicolumn{5}{|c|}{ South American Psychotria species } \\
\hline P. adderleyi Steyerm. & $2.5-4 \times 3.5-4$ & subglobose & 5 rounded & hsf \\
\hline P. adenophora Steyerm. & $5 \times 3$ & ovoid-oblong & 5 rounded & hsf \\
\hline P. amita Standl. & $4-5 \times 4-5$ & subglobose-oblong & $3-5$ rounded & hsf \\
\hline P. anceps HBK. & $5-6 \times 4.5-6$ & subglobose & 5 obscure & hsf \\
\hline P. anisopoda (Standl.) Steyerm. & $8 \times 4$ & ovoid-oblong & 4 obtuse & hsf \\
\hline P. apoda Steyerm. & $12 \times 15$ & subglobose & & hsf \\
\hline P. aristeguietae Steyerm. & $7-12 \times 6-10$ & subglobose-ovoid & $3-5$ rounded & hsf \\
\hline P. araguana Standl. & $3 \times 4$ & subglobose & 5 rounded & hsf \\
\hline P. aschersoniana Schum. et K. Krause & $4.5 \times 2.5$ & ovoid-oblong & 5 acute & hsf \\
\hline P. aubletiana Steyerm. & $4 \times 2.5$ & ovoid-oblong & 5 obtuse & hsf \\
\hline P. avilensis Steyerm. & $5 \times 4$ & subglobose & 5 obtuse & hsf \\
\hline
\end{tabular}


Table 1 (continued)

\begin{tabular}{|c|c|c|c|c|}
\hline Species & $\begin{array}{l}\text { Drupe size } \\
(\mathrm{mm})\end{array}$ & Shape & $\begin{array}{l}\text { Pyrene dorsal } \\
\text { ribs }\end{array}$ & $\begin{array}{l}\text { Sec- } \\
\text { tion }\end{array}$ \\
\hline P. bahiensis DC. & $5 \times 6$ & didymous & smooth & hsf \\
\hline P. blakei Standl. et Steyerm. & $4.5 \times 4$ & subglobose & 3 rounded & hsf \\
\hline P. blepharophora (Standl.) Steyerm. & $9 \times 5$ & ovoid-oblong & & hsf \\
\hline P. borjensis HBK. & $3.5-5 \times 3$ & ovoid-elliptical & 5 obtuse & hsf \\
\hline P. botryocephala (Standl.) Steyerm. & $5-6 \times 3-4$ & ovoid-oblong & 5 rounded & hsf \\
\hline P. bostrychothyrsus Sandw. & $3.5 \times 4.5-5$ & globose & 5 rounded & hsf \\
\hline P. bracteocardia (A. DC.) Müll.-Arg. & $3-4 \times 2-3$ & elliptical-ovoid & 5 rounded & hsf \\
\hline P. calciphila Steyerm. & $6-7 \times 4-6$ & subglobose-ovoid & 5 acute & hsf \\
\hline P. campylopoda Standl. & $4-5 \times 3$ & subglobose-obovoid & smooth & hsf \\
\hline $\begin{array}{l}\text { P. cardiomorpha C. M. Taylor et A. } \\
\text { Pool }\end{array}$ & $2-2.5 \times 3$ & subglobose & 3 obtuse & hsf \\
\hline P. carthagenensis Jacq. & $\begin{array}{l}4.5-6.5 \times \\
2.5-5\end{array}$ & oblong & 5 rounded & hsf \\
\hline P. celiae Steyerm. & $\begin{array}{l}4.5-6 \times \\
3.5-4.5\end{array}$ & elipsoidal & $4-5$ rounded & hsf \\
\hline P. ceratantha Standl. & $5-6 \times 6.5-10$ & subglobose & smooth & hsf \\
\hline $\begin{array}{l}\text { P. colorata (Willd. ex Roem. et } \\
\text { Schult.) Müll.-Arg. }\end{array}$ & $5-5.5 \times 3-3.5$ & ovoid-oblong & 5 obtuse & hsf \\
\hline P. concinna Oliver & $5 \times 4$ & subglobose-ovoid & $3-5$ acute & hsf \\
\hline $\begin{array}{l}\text { P. costularia (Baill.) Standl. et } \\
\text { Steyerm. }\end{array}$ & $5 \times 5$ & globose & $3-5$ acute & hsf \\
\hline P. coussareoides Standl. & $6-7 \times 6-6.5$ & subglobose & 2 obtuse & hsf \\
\hline P. crocochlamys Sandw. & $5-15 \times 3.5-12$ & ovoid-subglobose & 5 obtuse & hsf \\
\hline P. cuatrecasasii Steyerm. & $2-2.1 \times 1-1.2$ & oblong-elliptical & 5 obtuse & hsf \\
\hline P. cupularis (Müll.-Arg.) Standl. & $5-7 \times 3.5-5$ & subglobose-ovoid & 5 rounded & hsf \\
\hline P. duricoria Standl. et Steyerm. & $3-4 \times 4$ & subglobose & 5 rounded & hsf \\
\hline P. eciliata Steyerm. & $5-6 \times 4$ & ovoid & 5 obtuse & hsf \\
\hline P. eggersii Standl. & $4 \times 4$ & globose & 3 acute & hsf \\
\hline P. erecta (Aubl.) Standl. et Steyerm. & $8-10 \times 4.5-8$ & ovoid-oblong & smooth & hsf \\
\hline P. everardii Wernh. & $5 \times 5$ & subglobose & 5 rounded & hsf \\
\hline P. fanshawei (Standl.) Steyerm. & $11-13 \times 5-9$ & oblong-obovate & 5 obtuse & hsf \\
\hline P. fortuita Standl. & $7 \times 5-6$ & subglobose & $3-4$ obtuse & hsf \\
\hline P. franquevilleana Müll.-Arg. & $8 \times 6$ & subglobose-ovoid & 5 acute & hsf \\
\hline P. glandulicalyx Steyerm. & & subglobose-ovoid & $3-5$ rounded & hsf \\
\hline P. grandis Sw. & $5-6 \times 5-6$ & globose & 5 obtuse & hsf \\
\hline P. hemicephaelis Wernh. & $5-8 \times 5-8$ & subglobose & $4-5$ rounded & hsf \\
\hline
\end{tabular}


Table 1 (continued)

\begin{tabular}{|c|c|c|c|c|}
\hline Species & $\begin{array}{c}\text { Drupe size } \\
(\mathrm{mm})\end{array}$ & Shape & $\begin{array}{c}\text { Pyrene dorsal } \\
\text { ribs }\end{array}$ & $\begin{array}{l}\text { Sec- } \\
\text { tion }\end{array}$ \\
\hline P. heteroneura Steyerm. & $6 \times 6$ & subglobose & 4 rounded & hsf \\
\hline P. hyalina Steyerm. & $7 \times 4$ & ovoid & 5 rounded & hsf \\
\hline P. imthurniana Oliver & $3.5 \times 3.5$ & subglobose & 4 rounded & hsf \\
\hline P. iodotricha Müll.-Arg. & $12-13 \times 12-15$ & ovoid-subglobose & 4 rounded & hsf \\
\hline P. irwinii Steyerm. & $6-7.5 \times 5-7$ & ovoid-oblong & 5 obtuse & hsf \\
\hline P. jauensis Steyerm. & $4 \times 7$ & subglobose-ovoid & & hsf \\
\hline P. leiantha Steyerm. & $5.5 \times 4$ & globose-ovoid & 4 rounded & hsf \\
\hline P. lindenii Standl. & $3 \times 4$ & subglobose & 4 obtuse & hsf \\
\hline P. lupulina Benth. in Hook. & $5 \times 4$ & globose & smooth & hsf \\
\hline P. maguireorum Steyerm. & $4 \times 4$ & ovoid-subglobose & 5 acute & hsf \\
\hline P. mapourioides DC. & $5-7.5 \times 4-5$ & subglobose-ovoid & 5 obtuse & hsf \\
\hline P. marginata Sw. & $44 \times 3-3.5$ & subgobose & 5 rounded & hsf \\
\hline P. maturacensis Steyerm. & $13 \times 10$ & ovoid-oblong & 5 acute & hsf \\
\hline P. medusula Müll.-Arg. & $10 \times 0$ & globose & & hsf \\
\hline P. meridensis Steyerm. & $4-4.5 \times 2.5-3$ & ovoid-oblong & 5 acute & hsf \\
\hline P. micrantha HBK. & $5-5.5 \times 3-3.5$ & ovoid-oblong & 5 rounded & hsf \\
\hline P. microbotrys Ruiz ex Standl. & $33-3.5 \times 3-4$ & subglobose & 5 acute & hsf \\
\hline P. muscosa (Jacq.) Steyerm. & $4-7 \times 4-7$ & ellipsoid-subglobose & $4-5$ rounded & hsf \\
\hline P. nervosa Sw. & $5-7 \times 3-4.5$ & ovoid-oblong & 5 rounded & hsf \\
\hline P. oblita Wernh. & $6-8 \times 3-4$ & ellipsoidal & $4-5$ acute & hsf \\
\hline P. officinalis (Aubl.) Sandw. & $3 \times 3-5$ & globose-obovoid & & hsf \\
\hline P. pariensis Steyerm. & $5 \times 5$ & subglobose & 5 obtuse & hsf \\
\hline P. parvibractea Steyerm. & $4 \times 3$ & subglobose-ovoid & $3-5$ acute & hsf \\
\hline P. phaneroloma Standl. et Steyerm. & $2 \times 2.8$ & subglobose & & hsf \\
\hline P. platypoda A. DC. & $2-4 \times 3.5-4$ & subglobose & 5 obtuse & hsf \\
\hline P. polycephala Benth. in Hook. & $2 \times 2.5-3.5$ & subglobose & $\begin{array}{c}3-5 \text { verrucu- } \\
\text { lose }\end{array}$ & hsf \\
\hline P. prunifolia (HBK.) Steyerm. & $5-7 \times 3-4$ & ovoid-oblong & & hsf \\
\hline P. psychotriifolia (Seem.) Standl. & $8-9 \times 4-6$ & oblong & 5 obtuse & hsf \\
\hline P. racemosa (Aubl.) Raeusch. & $3.5-4 \times 5-6$ & subglobose & 5 obtuse & 3-ang \\
\hline P. remota Benth. in Hook. & $5-6 \times 3-4$ & ellipsoidal & $4-5$ rounded & hsf \\
\hline P. rosacea Steyerm. & $7 \times 5$ & ellipsoidal & 5 rounded & hsf \\
\hline P. rosea (Benth.) Müll.-Arg. & $5 \times 4.5$ & subglobose-ovoid & 5 rounded & hsf \\
\hline P. ruiz-terani Steyerm. & $6 \times 6$ & globose & 5 rounded & hsf \\
\hline P. santamartensis Rusby & $5-6.5 \times 5-7$ & & & \\
\hline
\end{tabular}


Table 1 (continued)

\begin{tabular}{|c|c|c|c|c|}
\hline Species & $\begin{array}{l}\text { Drupe size } \\
(\mathrm{mm})\end{array}$ & Shape & $\begin{array}{l}\text { Pyrene dorsal } \\
\text { ribs }\end{array}$ & $\begin{array}{l}\text { Sec- } \\
\text { tion }\end{array}$ \\
\hline P. schomburgkii Benth. in Hook. & $4-4.5 \times 3$ & oblong-elliptical & $3-5$ rounded & hsf \\
\hline $\begin{array}{l}\text { P. spadicea (Pittier) Standl. et } \\
\text { Steyerm. }\end{array}$ & $3 \times 3$ & subglobose-obovoid & 5 obscure & hsf \\
\hline P. speluncae Standl. et Steyerm. & $6-8 \times 6,5-7$ & subglobose & $3-5$ acute & hsf \\
\hline P. sphaerocephala Müll.-Arg. & $3-4 \times 2.5-3$ & subglobose & 5 obtuse & hsf \\
\hline P. stipulosa Müll.-Arg. & $5 \times 4.5$ & ovoid-subglobose & $3-5$ acute & hsf \\
\hline P. subundulata Benth. in Hook. & $2 \times 3$ & subglobose & 5 obtuse & hsf \\
\hline P. tepuiensis (Steyerm.) Steyerm. & $8-15 \times 10-13$ & subglobose & 5 acute & hsf \\
\hline P. transiens Wernh. & $15 \times 10$ & ovoid-subglobose & 5 obtuse & hsf \\
\hline P. trichotoma Mart. et Gal. & $7-10 \times 5-7$ & obovoid & 5-7 rounded & hsf \\
\hline P. ulviformis Steyerm. & $4-9 \times 4-7$ & subglobose & smooth & hsf \\
\hline P. variegata Steyerm. & $4-7 \times 2-7$ & subglobose & smooth & hsf \\
\hline P. vasivensis (Müll.-Arg.) Standl. & $7-9 \times 4.5-7$ & oblong-elliptical & & \\
\hline P. vichadensis Standl. & $2 \times 2$ & globose & $3-5$ rounded & hsf \\
\hline P. yaracuyensis Steyerm. & $6.5 \times 5$ & subglobose & 5 rounded & hsf \\
\hline \multicolumn{5}{|c|}{ South American Palicourea species } \\
\hline P. acetosoides Wernh. & $4-5 \times 3-3.5$ & ovoid & 5 rounded & hsf \\
\hline P. angustifolia Kunth in HBK. & $3-4 \times 4-5$ & subglobose & $4-5$ rounded & hsf \\
\hline P. apicata in HBK. & $5-8 \times 5-7.5$ & subglobose & $3-4$ rounded & hsf \\
\hline P. aschersonioides (Wernh.) Steyerm. & $8 \times 8$ & subglobose & 5 rounded & hsf \\
\hline P. calophylla DC. & $6-7 \times 5-6$ & subglobose & $4-5$ acute & hsf \\
\hline P. canaguensis Steyerm. & $10 \times 7$ & ovoid & $4-5$ rounded & hsf \\
\hline P. corymbifera (Müll.-Arg.) Standl. & $3 \times 4$ & subglobose & 5 rounded & hsf \\
\hline P. ctenocalyx Steyerm. & $7 \times 5-7$ & subglobose & 5 rounded & hsf \\
\hline P. demissa Standl. & $8-9 \times 7-8$ & ovoid & smooth & hsf \\
\hline P. dunstervilleorum Steyerm. & $3-4 \times 4-5$ & globose & 4 rounded & hsf \\
\hline P. flexiramea Steyerm. & $5-6 \times 4-5$ & subglobose-ovoid & 5 obtuse & hsf \\
\hline P. garciae Steyerm. & $5 \times 5-7$ & globose & $4-5$ rounded & hsf \\
\hline P. grandiflora (Kunth) Standl. & $5-7 \times 6-7$ & subglobose & $4-5$ rounded & hsf \\
\hline $\begin{array}{l}\text { P. grandifolia (Willd. ex Roem. et } \\
\text { Schult.) Standl. }\end{array}$ & $4 \times 4$ & subglobose & 5 rounded & hsf \\
\hline P. huntingii Steyerm. & $4 \times 4$ & subglobose & 5 acute & hsf \\
\hline P. insignis Steyerm. & $5 \times 5-7$ & globose & 4 rounded & hsf \\
\hline P. jahnii Standl. & $3-4 \times 3.5-4$ & subglobose & 3-4 obtuse & hsf \\
\hline P. lancigera (Standl.) Steyerm. & $4 \times 3-3.5$ & elliptical & $4-5$ rounded & hsf \\
\hline
\end{tabular}


Table 1 (continued)

\begin{tabular}{lcccc}
\hline Species & $\begin{array}{c}\text { Drupe size } \\
(\mathrm{mm})\end{array}$ & Shape & $\begin{array}{c}\text { Pyrene dorsal } \\
\text { ribs }\end{array}$ & $\begin{array}{c}\text { Sec- } \\
\text { tion }\end{array}$ \\
\hline P. leuconeura Standl. & $3 \times 2-2.5$ & elliptical-subglobose & $4-5$ acute & hsf \\
P. longiflora DC. & $4.5-5 \times 5$ & subglobose & 5 obtuse & hsf \\
P. longistipulata Standl. & $4.5-5 \times 3-4$ & subglobose-ovoid & $4-5$ obtuse & hsf \\
P. meridensis Steyerm. & $5 \times 4$ & elliptical & 5 rounded & hsf \\
P. nitidella (Müll.-Arg.) Standl. & $4 \times 3-3.5$ & subglobose & 5 rounded & hsf \\
P. nubicola Steyerm. & $8 \times 6-7$ & ovoid & $4-5$ obtuse & hsf \\
P. obtusata K. Krause & $4-5 \times 2.5-3$ & ovoid & & hsf \\
P. perquadrangularis Wernh. & $3-3.5 \times 3-3.5$ & globose & $4-5$ acute & hsf \\
P. petiolaris Kunth in HBK. & $3.5-4 \times 3$ & elliptical-subglobose & $4-5$ rounded & hsf \\
P. pittieri Standl. & $5-5.5 \times 3.5-4$ & ovoid & 4 obtuse & $\mathrm{hsf}$ \\
P. puberulenta Steyerm. & $10-13 \times 10-13$ & globose & 5 obtuse & $\mathrm{hsf}$ \\
P. rigida HBK. & $5-6 \times 4-5$ & subglobose & 5 obtuse & $\mathrm{hsf}$ \\
P. semirasa Standl. & $5-6 \times 4.5-6$ & subglobose-ovoid & 4 obtuse & $\mathrm{hsf}$ \\
P. tamaensis (Standl. et Steyerm.) & $7 \times 5$ & subglobose-ovoid & 4 rounded & $\mathrm{hsf}$ \\
Steyerm. & $5 \times 4$ & ovoid & 5 rounded & $\mathrm{hsf}$ \\
P. tepuicola Steyerm. & $5-6 \times 5$ & subglobose-ovoid & 5 acute & $\mathrm{hsf}$ \\
P. thyrsiflora (Ruiz et Pav.) DC. & & & &
\end{tabular}

itly 4-angular, and embedded in a fleshy mesocarpium. The fruits contain 2 pyrenes, which are triqueter, the lateral sides are curvous and the dorsal angle forms an elevated crest bordered by two marginal ribs and a channel between, which may serve as alternative or secondary germination slit (Fig. 3, right); the 2 lateral angles are bordered by irregularly crenulated marginal ribs. The cross section of the pyrene is plane-concave 3-angular. The ventral surface is flat and smooth with a central longitudinal line through introduces the endocarp into forming a keel; the germination hole is located at the basal end.

Palicourea tetragona is distributed from Mexico to Panama and P. seemannii from Nicaragua to Ecuador.

The above listed differences were neglected in recent monographical treatments (Taylor 2012a, b. Taylor et al. 2010). Since fruit and pyrene morphology are important for the distinction of different genera, our proposal is to separate the above species and erect a new genus or genera.

The two questions are whether the two species Palicourea seemannii and $P$. tetragona are closely related and belonging to one genus, or they are very different representing two monotypic genera? The following key gives an answer to these questions, listing their most important distinctive characters in relation to the two large genera where they were formerly placed. 
Identification key to the related genera

1a Drupes with 6-10 longitudinal ribs or sometimes smooth surface; pyrenes 2 (5) plano-convex, with 3-5 adaxial (dorsal) ribs; cross section hemispherical or obtriangular

$1 \mathrm{~b}$ Drupes 4-angulate to 4-costate, pyrenes 2, triqueter with an elevated dorsal-central crest and 2 lateral flattened lobes with slightly thickened, irregularly crenulated margin; cross section triangular

2a Corolla tube elongate, more or less curved, gibbous, with a hairy ring inside at or above the swelling; inflorescences reddish or yellowish, corolla usually bright yellow to red

Palicourea subgen. Palicourea

$2 \mathrm{~b}$ Corolla tube straight, not gibbous; hairy ring inside above the middle of the tube, or naked, inflorescence green or white, flowers white, pale yellow or greenish

3a Stipules usually sheathed, entire, rounded or hood-shaped at the apex, sometimes bi-aristate, brown or ferrugineous, deciduous soon leaving a ring scar covered by a fringe of reddish brown hairs; leaves drying black, greyish or reddish; inflorescences green, mature fruit red Psychotria

3b Stipules usually bilobated or bidentate, green, persistent; leaves drying green, greyish or yellowish green to yellowish brown; inflorescence usually white, mature fruit blue, black or purple

Palicourea subgen. Heteropsychotria

4a Inflorescence paniculate, green to blue or violet; flowers homostylous, pedicellate, with short triangular bracts; calyx 5-lobulated; corolla blue or purple, tube gibbous at the base, puberulent outside and villous inside with two hairy rings, one above the swollen base, and the second faucial, emerging from the throat; stamens sessile or subsessile placed in the naked central part of the tube, between the two hairy rings, anthers dorsifixed near the base; pistil capitate or bidentate with two short orbicular stigmas

Ditrichanthus

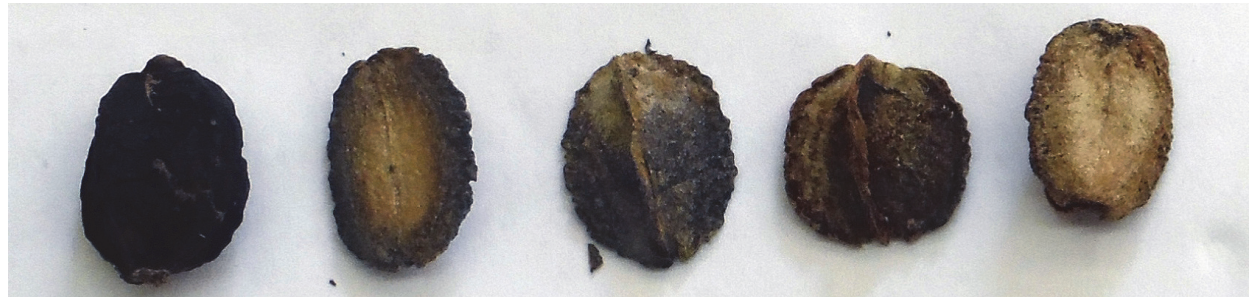

Fig. 2. The form of pyrenes on the genus Mexocarpus, from selected seeds of fruiting specimens of Mexocarpus tetragonus of MEXU (photo: A. Borhidi) 
Table 2

Main distinctive features of the treated taxonomic units

\begin{tabular}{|c|c|c|c|c|c|}
\hline $\begin{array}{l}\text { Genus, } \\
\text { subgenus }\end{array}$ & $\begin{array}{l}\text { Psychotria } \\
\text { s. str. }\end{array}$ & $\begin{array}{c}\text { Palicourea } \\
\text { subgen. } \\
\text { Hetero- } \\
\text { psychotria }\end{array}$ & $\begin{array}{l}\text { Palicourea } \\
\text { subgen. } \\
\text { Palicourea }\end{array}$ & Ditrichanthus & $\begin{array}{l}\text { Mexocar- } \\
\text { pus }\end{array}$ \\
\hline Fruit & 6-10-ribbed & 6-10-ribbed & 6-10-ribbed & 4-angulate & 4-angulate \\
\hline $\begin{array}{l}\text { Number of } \\
\text { pyrenes }\end{array}$ & $2(-5)$ & $2(-5)$ & $2(-5)$ & 2 & 2 \\
\hline $\begin{array}{l}\text { Form of } \\
\text { pyrenes }\end{array}$ & $\begin{array}{l}\text { Plano-con- } \\
\text { vex }\end{array}$ & $\begin{array}{l}\text { Plano-con- } \\
\text { vex }\end{array}$ & $\begin{array}{l}\text { Plano-con- } \\
\text { vex }\end{array}$ & Triqueter & Triqueter \\
\hline $\begin{array}{l}\text { Colour of } \\
\text { inflorescence }\end{array}$ & Green & White & $\begin{array}{l}\text { Reddish- } \\
\text { yellow }\end{array}$ & $\begin{array}{l}\text { Green-blue- } \\
\text { violet }\end{array}$ & Green \\
\hline $\begin{array}{l}\text { Form of co- } \\
\text { rolla tube }\end{array}$ & Straight & Straight & $\begin{array}{l}\text { Curved- } \\
\text { gibbous }\end{array}$ & $\begin{array}{l}\text { Straight- } \\
\text { gibbous }\end{array}$ & Straight \\
\hline $\begin{array}{l}\text { Hairyness in- } \\
\text { side the corolla }\end{array}$ & $\begin{array}{l}\text { In the mid- } \\
\text { dle or naked }\end{array}$ & $\begin{array}{l}\text { In the mid- } \\
\text { dle }\end{array}$ & $\begin{array}{l}\text { In the swell- } \\
\text { ing or above }\end{array}$ & $\begin{array}{l}\text { Two rings in } \\
\text { the swelling }\end{array}$ & $\begin{array}{l}\text { In the up- } \\
\text { per third }\end{array}$ \\
\hline $\begin{array}{l}\text { Colour of ma- } \\
\text { ture fruits }\end{array}$ & Red & $\begin{array}{c}\text { Blue-black- } \\
\text { purple }\end{array}$ & Blue-black & Blue-violet & $\begin{array}{l}\text { Purple- } \\
\text { black }\end{array}$ \\
\hline $\begin{array}{l}\text { Colour of the } \\
\text { corolla }\end{array}$ & $\begin{array}{l}\text { White-yel- } \\
\text { low-green }\end{array}$ & Yellow-red & $\begin{array}{l}\text { Yellow-red- } \\
\text { blue-violet }\end{array}$ & Blue-purple & White \\
\hline $\begin{array}{l}\text { Form of } \\
\text { stipules }\end{array}$ & $\begin{array}{l}\text { Sheathed } \\
\text { entire }\end{array}$ & $\begin{array}{l}\text { Bilobate- } \\
\text { bidentate }\end{array}$ & $\begin{array}{c}\text { Tubular } \\
\text { bidentate }\end{array}$ & $\begin{array}{c}\text { Tubular } \\
\text { lignescent }\end{array}$ & $\begin{array}{c}\text { Entire } \\
\text { triangular }\end{array}$ \\
\hline
\end{tabular}

$4 \mathrm{~b}$ Inflorescence cymose-paniculate, trichotomous, green, composed of 1-6 flowered glomerulate cymes surrounded by involucral bracts; flowers distyl, sessile; calyx tubular, truncate with undulate or denticulate margin; corolla white, tube straight, not gibbous at the base, naked inside except the upper third below the throat; stamens inserted below the hairy faucial ring, anthers dorsifixed in the middle, versatiles, emerging in the brevistyle form; style hairy, stigma bilobate with long branches Mexocarpus

\section{Mexocarpus Borhidi, E. Martínez et Ramos, genus novum, hoc loco} (sect. Palicoureeae)

Type species: Cephaëlis tetragona Donn.-Sm., Bot. Gaz. 61: 376 (1916). Type specimen: Costa Rica. Cartago, forets de Tuis, 650 m, Oct. 1897, A. Tonduz 11352.

Arbor usque ad $30 \mathrm{~m}$ alta vel frutices $2.5 \mathrm{~m}$ alti. Stipulae laminares persistentes, coriaceae, cum aetate lignescentes et horizontaliter extensae, integrae vel breviter excisae, saepe dorso carinatae, glabrae. Folia petiolata, chartacea, concolor, in sicco viridis vel cinerascens, margine integra. Inflorescentiae 3-radiales, terminales, cymoso- 
paniculatae, rami 4-anguati vel compressi, breviter ferrugineo-pubescentes in 2 latis oppositis, flores dichostyles in capitulis sessilibus 1-6-floris bracteolis 3-4 foliaceis brevibus involucrati. Hypanthium obovatum vel subglobosum, obscure 4-angulatum, limbus calycis tubularis margine undulatus vel irregulariter denticulatus, sine lobulis bene formatis, utrinque glabris. Corolla alba hipocraterimorfa 25-45 mm longa, tubus 20-35 mm longus, intus basi glaber, tertio superiori hirsutus vel villosus, lobi (4-)5, oblongo-ovati vel lanceolati, 9-15 $\mathrm{mm}$ longi et 3-4 mm lati, apice acuti incrassati et sub anthesi reflexi, basi adaxialiter sericeo-pilosi, ceterum utrinque glabri, stamina 5, sub fauce affixa, filamenta glabra, antherae lineares, cerca dimidium dorsifixae, versatiles, in floribus brevistylis e fauce leviter exsertae in floribus longistylis in tubo sub fauce inclusae, stylus supra dimidium patenter pilosus, stigma bilobatum. Fructus drupaceus, subglobosus, elipsoideus vel obovatus, 10-16 mm longus et 9-13 mm latus, 4-angulatus, tubo calycis coronatus, pyrenae 2, 3-lobata in sectione transversali concavio-triangularia, crista centrali emergenti, lateralibus margine leviter incrassatis et irregulariter crenulatis. Semen oblong-ellipticum, ventraliter canaliculatum.

\section{Mexocarpus tetragonus (Donn.-Sm.) Borhidi, E. Martínez et Ramos, comb. nova, hoc loco}

(Figs 2-9)

Bas.: Cephaëlis tetragona Donn.-Sm., Bot. Gaz. 61: 376 (1916) झ Palicourea tetragona (Donn.Sm.) C. M. Taylor, Novon 20: 490 (2010), non Psychotria tetragona Seem., 1867. - Syn.: Psychotria chiapensis Standl., Contr. U. S. Natl. Herb. 23: 1390 (1926).

Shrubs, small or large trees up to $30 \mathrm{~m}$ tall, trunk $20-50 \mathrm{~cm}$ diameter, bark smooth, much-branched with rounded crown, leafy stems $2-8 \mathrm{~mm}$ thick, terete, often contracted below the node after drying, glabrous or with short hairs in new growth. Stipules laminar, persistent, coriaceous, thickened and ligneous with age, $2-5 \mathrm{~mm}$ long, 2-4(-8) $\mathrm{mm}$ broad, broadly triangular to ovate, rounded or acute, entire or with a small apical sinus with 2 triangular

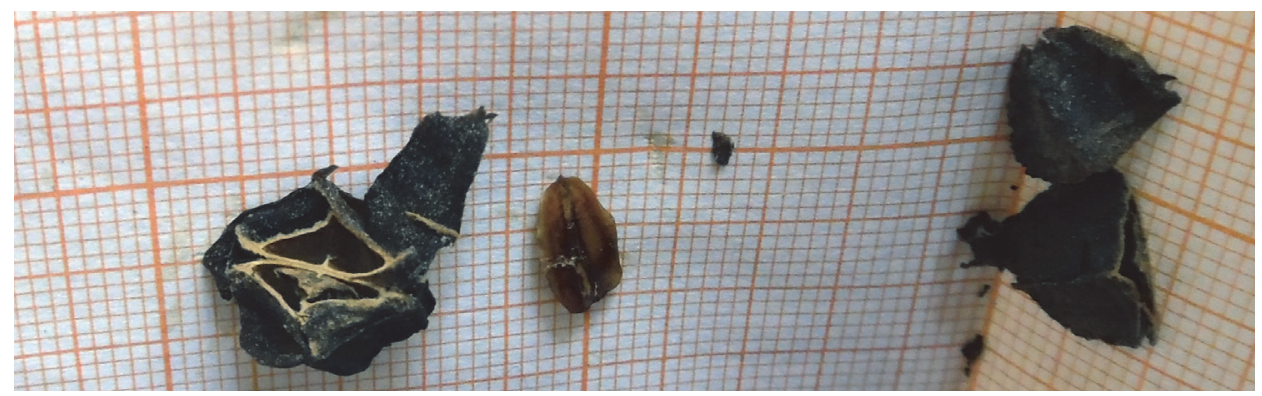

Fig. 3. Cross section of a pyrene of Mexocarpus tetragonus (Aguilar y Arcos 11455, MEXU) (photo: A. Borhidi) 
lobes to $2 \mathrm{~mm}$ long, often with a strong white dorsal nerve, glabrous on both sides (Fig. 6), or very short puberulent abaxially, with 2 groups of ferrugineus hairs at the basal adaxial angles, green when young, later brownish to blackish, spoon-like and horizontally extended (Fig. 7). Leaves with petioles, 30-35
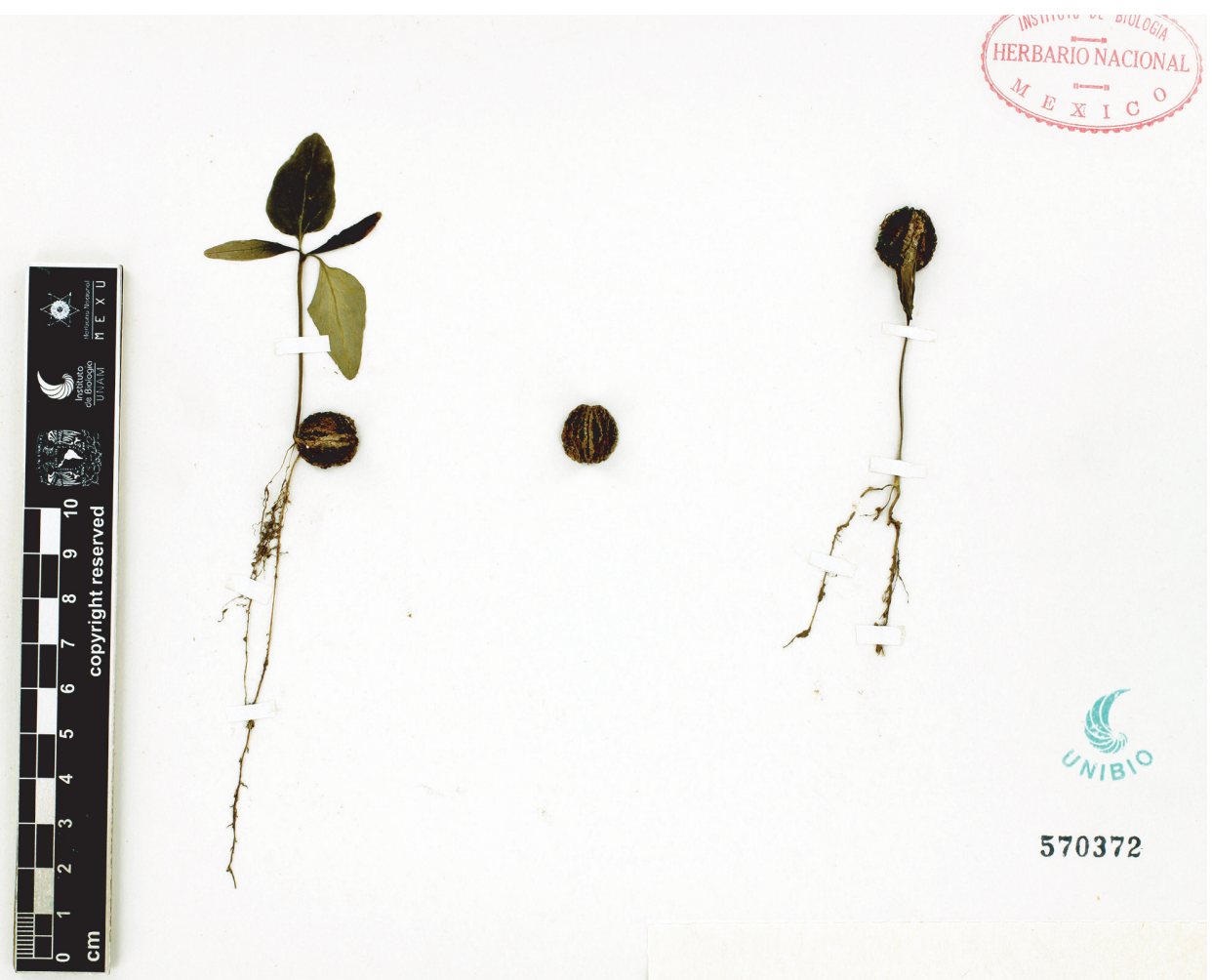

570372

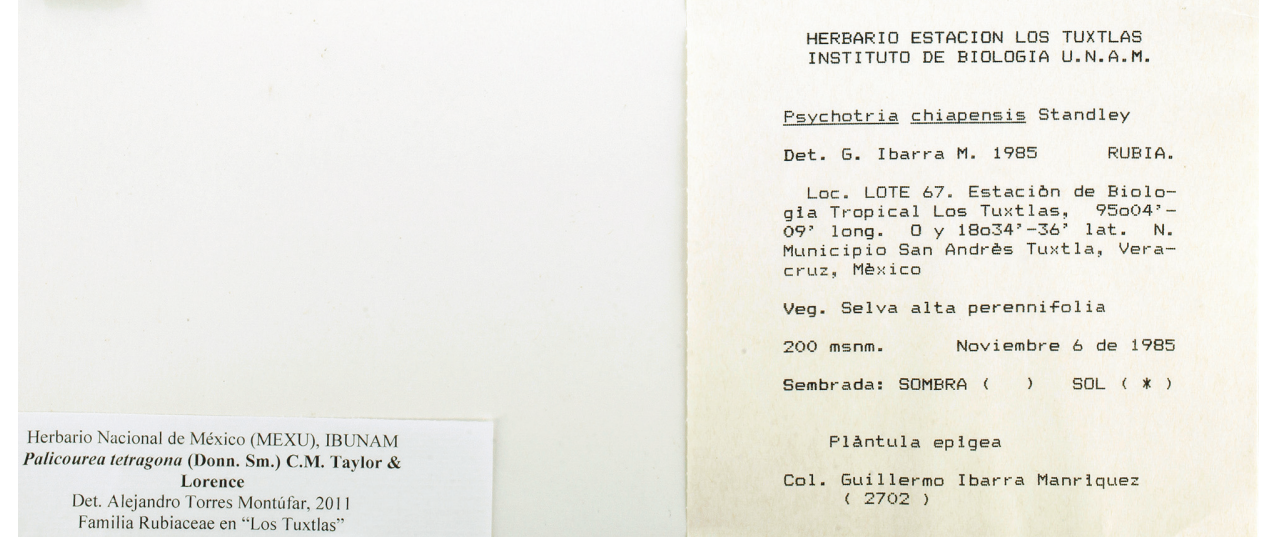

Fig. 4. Germination of Mexocarpus tetragonus pyrenes (G. Ibarra, M. 2702, MEXU) 
$\mathrm{mm}$ long and $0.8-2.5 \mathrm{~mm}$ thick, glabrous or sometimes puberulent near the inflorescence; blades $2.5-2.8 \mathrm{~cm}$ long and $10-11.5 \mathrm{~mm}$ broad, elliptical-obovate, to elliptical-oblong or narrowly oblong-obovate, short-apiculate or acute at the apex with tip 3-10 mm long, and gradually narrowed to a cuneate base, slightly decurrent on petiole, drying thin-chartaceous and greyish green to dark brown above, paler beneath, glabrous above and below except for short hairs on the nerves beneath; lateral veins 5-13 pairs prominent with the intersecondary ones, reticulation transparent till the quaternary nerves on both sides; margin thin, entire, glabrous. Inflorescence terminal, 3-radial, cymosecorymbose $4-10 \mathrm{~cm}$ long, $5-8 \mathrm{~cm}$ broad, with 3-7 bracteolate flower clusters, peduncles compressed, 1-8-15 cm long, puberulent, bracts 2, euphylloid with petioles 1-6 mm long, blades elliptical-lanceolate, $3-5 \mathrm{~cm}$ long and $1.2-1.5 \mathrm{~cm}$ broad, principal axes 1.5-2 cm long, compressed or 4-angulate, with 2 lines short ferrugineous-pubescent on the opposite sides or glabrescent, terminal branches ending in 1-6-flowered heads encircled by an involucrum formed by 3-4 involucral bracteoles \pm connate at the base. Flowers distylous, sessile, hypanthium obovate, $0.5-1 \mathrm{~mm}$ long, acute at the base, ferrugineous-puberulent or glabrous. Calyx limb cylindrical, $4-6 \mathrm{~mm}$ long, $2-2.5 \mathrm{~mm}$ broad, glabrous on both sides truncate at the apex with 5 distant triangular denticles, persistent on the fruit. Corolla white, salverform, 25-45 mm long, tube 20-35 $\mathrm{mm}$ long, 2-3 mm broad, thickened, sericeo-pilose adaxially at the base or glabrous, hirsute or villous in the upper tertiary inside till the throat, lobes (4-)5 oblong-ovate or lanceolate, acute or obtuse, 9-15 $\mathrm{mm}$ long, 2-3 $\mathrm{mm}$ broad, thickened, reflexed, sericeo-pilose adaxially in the base, other parts glabrous. Stamens 4 or 5, filaments slender, $0.8-4 \mathrm{~mm}$ long, affixed $6-8 \mathrm{~mm}$ below the throat, anthers linear, dorsifixed in the middle, $4-5.5 \mathrm{~mm}$ long, $0.2-0.3 \mathrm{~mm}$ broad, enclosed in the longistyle form (Fig. 5D), versatile and slightly exerted in the brevistyle form (Fig. 8), style 30-35 mm long in the brevistyle form, $35-45 \mathrm{~mm}$ long in the longistyle form, hirsute with extended hairs above the middle, stigma bilobate with two linear, 3-4 mm long branches. Fruit globose (Fig. 9) or broadly elliptical, 4-angulate, 4-costate when dry, sometimes 6-costate when the costa become double in the commissure place, $10-16 \mathrm{~mm}$ long and $9-13 \mathrm{~cm}$ broad, crowned by the persistent calyx tube. Pyrenes 2 , triqueter, triangular-concave in transversal section with an elevated central crest and two marginal ribs on the top, lateral lobes thickened at the margin, adaxial side plate. Seeds smooth at the dorsal side, with a central longitudinal slit on the ventral side. The germination hole is located at basal end.

Specimens studied: Mexico: Chiapas: Mpio. Cintalapa, Emilio Rabasa, S. Ochoa-Gaona 3655 (MEXU); Mpio. Palenque. 15-20 km towards Chancala on road to Bonampak from the Palenque-Ocosingo road, D. E. Breedlove 49105 (MEXU); Mpio. Las Margaritas, Maravilla Tenejapa, Alush Mendez G. 9076 (MEXU); Mpio. Ocosingo, Bonampak, J. P. Abas- 


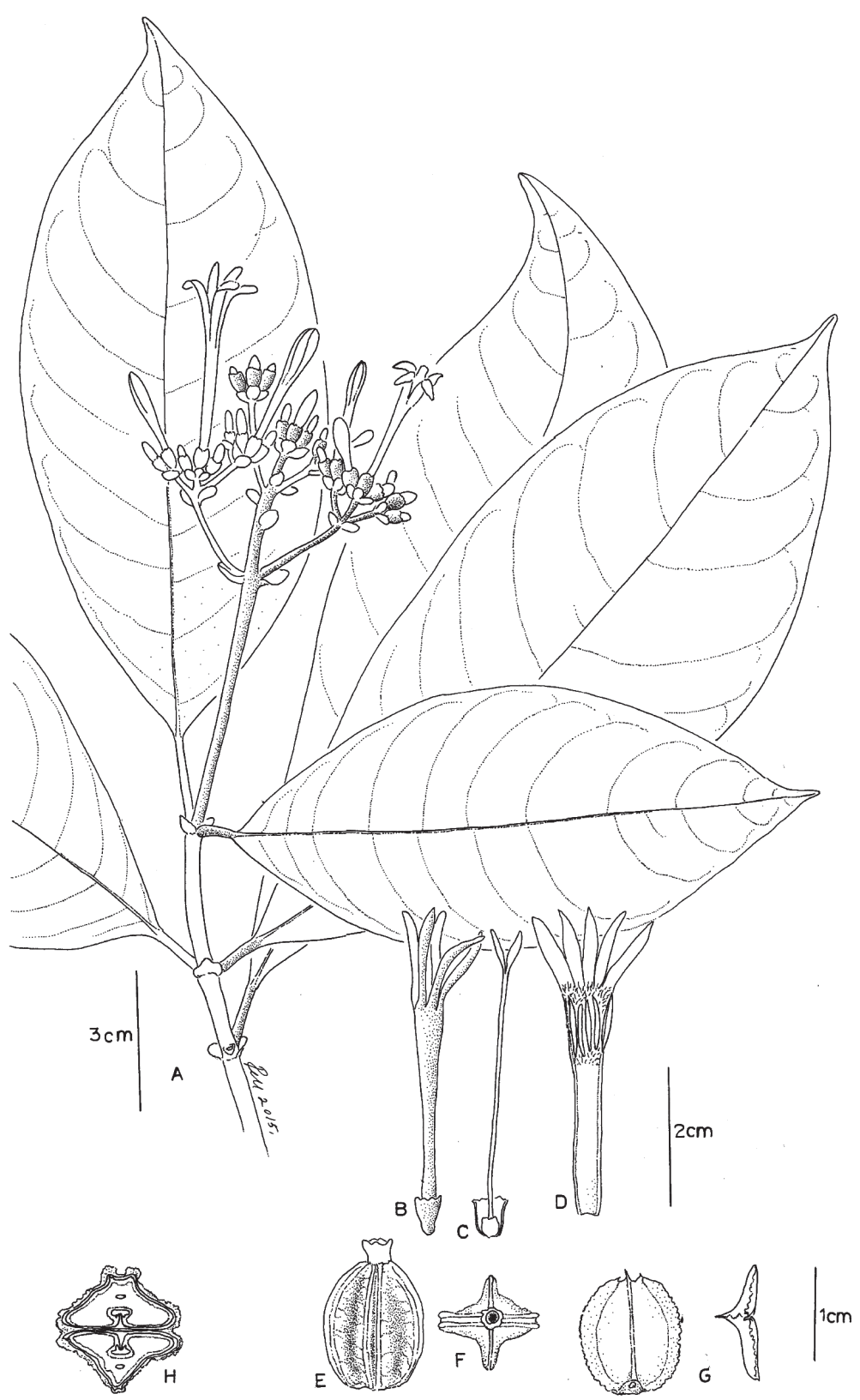

Fig. 5. Mexocarpus tetragonus (Donn.-Sm.) Borhidi, E. Martínez et Ramos. A = twigs with inflorescence, $\mathrm{B}=$ flower, $\mathrm{C}=$ calyx with pistil, $\mathrm{D}=$ corolla, longitudinal section with stamens (A-D: G. Aguilar 10695, MEXU), E = fruit, lateral view, F = fruit, apical view, $G=$ pyrene, ventral and apical view, $2 \times, \mathrm{H}=$ cross section of fruit $(\mathrm{E}-\mathrm{H}$ : G. Aguilar y Arcos 11455, MEXU) (drawings by M. en C. Ramiro Cruz Durán) 


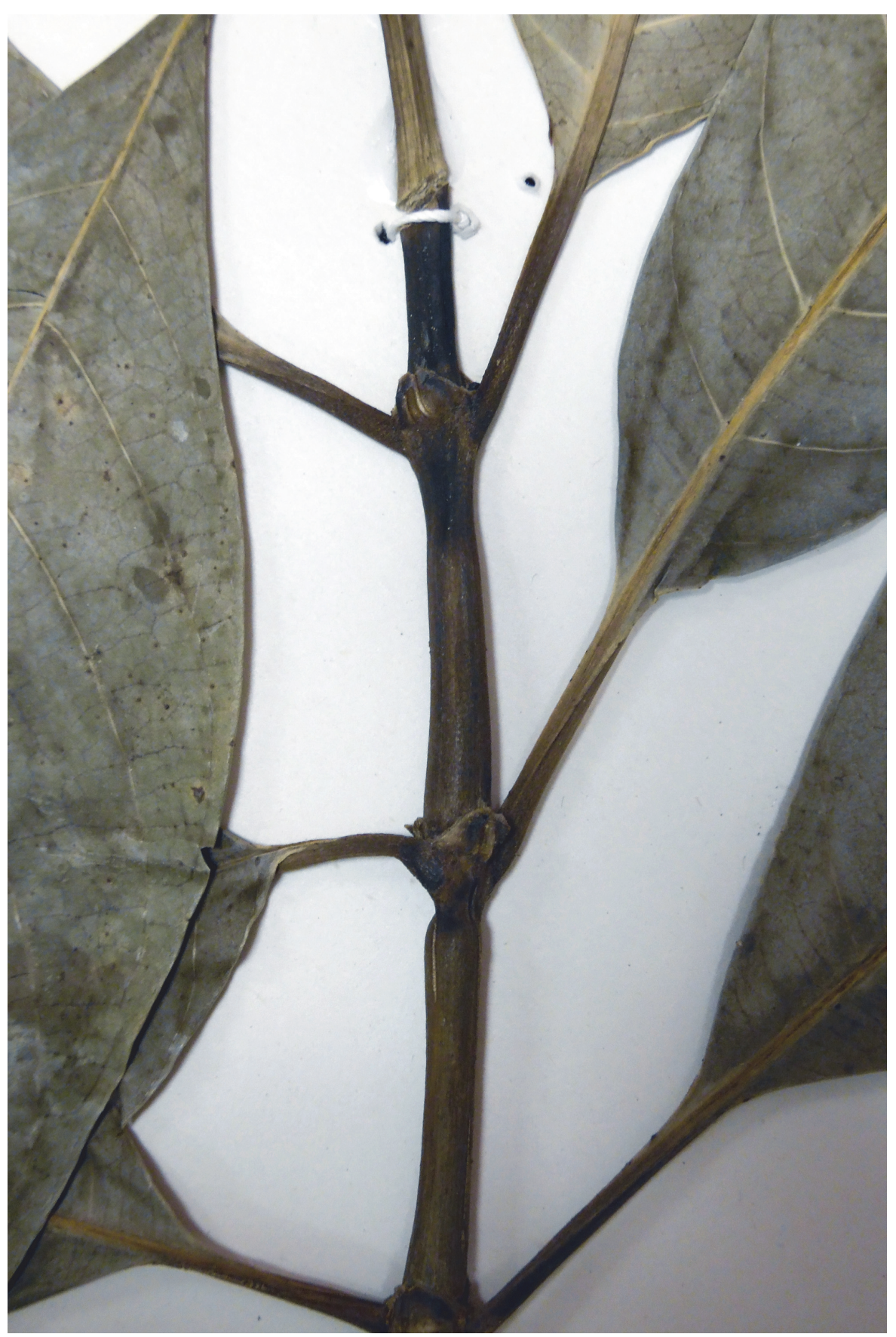

Fig. 6. Mexocarpus tetragonus, twig with young stipules (E. Martínez 12210, MEXU) (photo: A. Borhidi) 


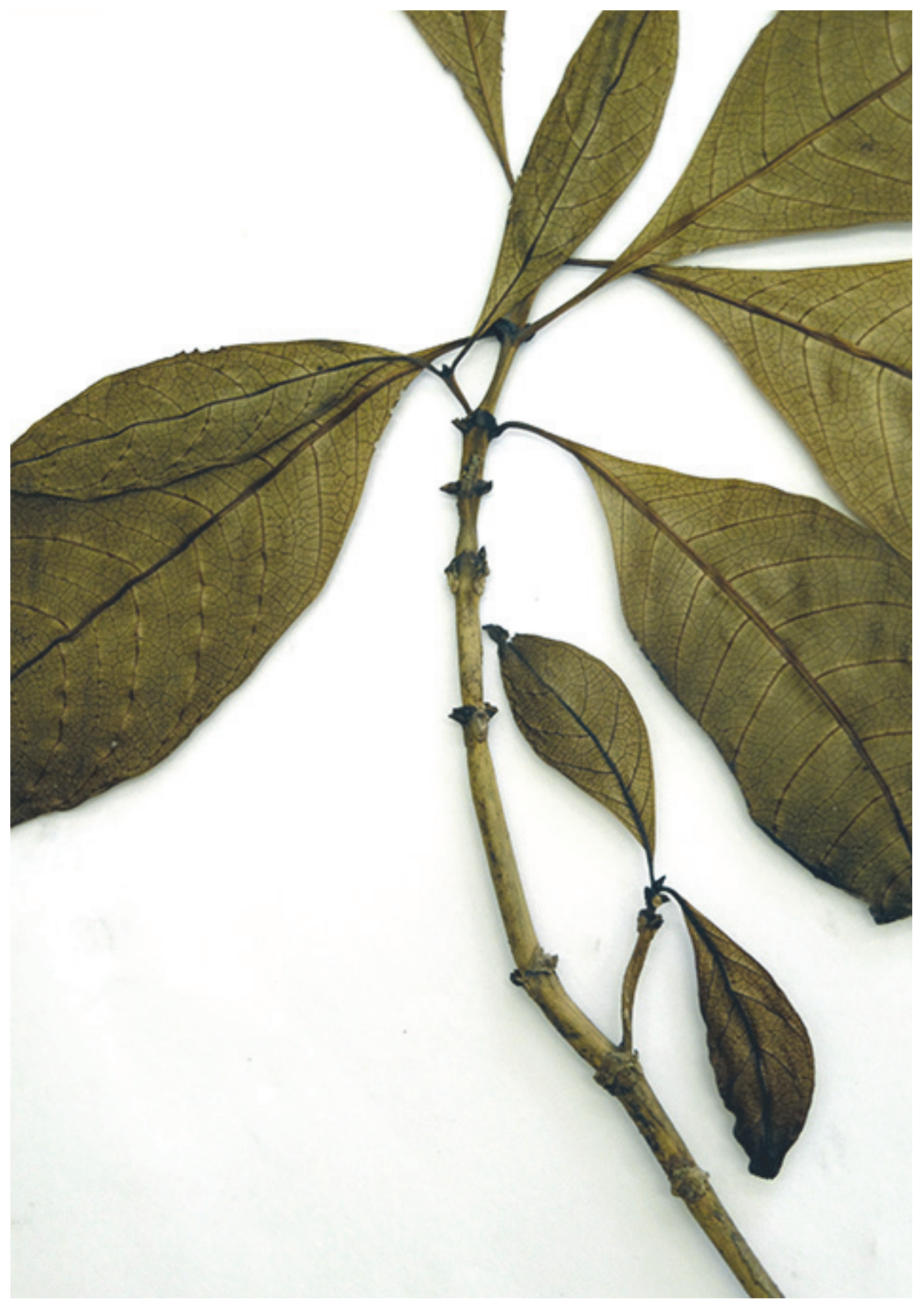

Fig. 7. Mexocarpus tetragonus, twig with aged stipules (G. Juárez 3675, MEXU) (photo: A. Borhidi) 
cal, 380 (MEXU); D. Álvarez, 3755 (MEXU); Laguna Lacanha, J. P. Abascal 287 (MEXU); Chansayab, G.Aguilar 3855 (MEXU); Comunidad Lacandona de Lacanha-Chansayab, G. Aguilar 7787 (MEXU); S. Levy \& A. Durán 156 (MEXU); M. González-Espinosa et al. 776 (MEXU); 1106 (MEXU); Frontera Corozal, G. Aguilar, 11477 (MEXU); 10315 (MEXU); 10350 (MEXU); 10048 (MEXU); E. Martínez S. 10876 (MEXU); 12299 (MEXU); 14595 (MEXU); 15463 (MEXU); 15592 (MEXU); San Javier, G. Aguilar 5656 (MEXU); J. Calónico 25229 (MEXU); Ejido Benemérito de las Américas, E. Martínez S. 7157 (MEXU), 9401 (MEXU); Reserva Montes Azules, G. Domínguez-Vázquez 429 (MEXU); Boca Lacantun, E. Martínez S. 7621 (MEXU); 18996 (MEXU); Arroyo Puerquito, E. Martínez S, 13669 (2) (MEXU); Rio Chixoy, E. Martínez S. 15918 (MEXU); A 5.53 km al NO del Crucero San Javier, D. Álvarez 6251 (MEXU); A 3 km al NE de San Javier, G. Aguilar 4066 (MEXU); Nuevo Jerusalén, G. Aguilar 3392 (MEXU); A 1.3 km al S de El Paraíso, G. Aguilar 3650 (MEXU); Mpio.

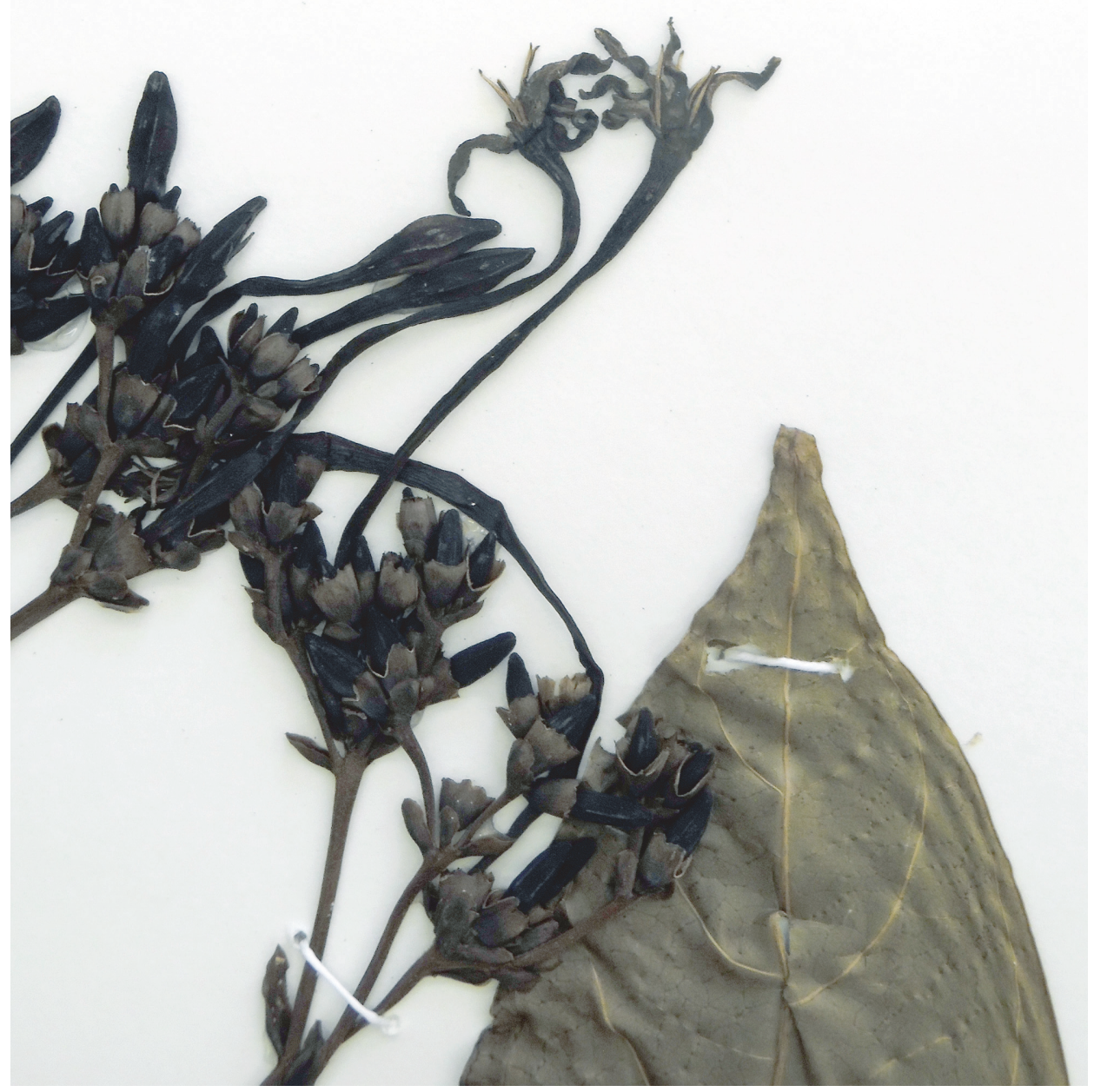

Fig. 8. Mexocarpus tetragonus, brevistyle flowers (E. Martínez 12210, MEXU) (photo: A. Borhidi) 
Palenque, Rio Chancalá, M. González-Espinosa et al. 862 (MEXU); 3 km al S del entronque a Chancala, T. Wendt et al. 2326 (MEXU); Mpio. Tapachula, Real Fer, E. Ventura \& E. López 116 (MEXU); Oaxaca, Distrito: Juchitán, Mpio. Guichicovi. Boca del Monte, G. Juárez 3675 (MEXU); Tabasco: Mpio. Teapa, Sierra de Madrigal, A. M. Hanan, 488 (MEXU); Mpio. Tenosique, a ca. $15 \mathrm{~km}$ arriba de La Palma, por río a $5 \mathrm{~km}$ del rancho Punta de la Montaña del Sr. Angel Zubieta, C. Cowan 3376 (MEXU); Veracruz: Mpio. Catemaco, 8 km al N de Catemaco, Col.: J. H. Beaman 6170 (MEXU); Mpio. Hidalgititlán, Brecha Hnos. Cedillo, La Laguna, $17^{\circ} 16^{\prime} \mathrm{N}, 94^{\circ} 37^{\prime} \mathrm{W}$, alt. $200 \mathrm{~m} \mathrm{snm}$. Selva alta perennifolia, secundaria, suelo rojizo algo profundo sobre calizas. Árbol $5 \mathrm{~m}$ fruto verde. Col.: Brigada Vazquez 2, 14.02.1974. No. 40474 (MEXU); Mpio Las Choapas. Ceiba Blanca, J. Dorantes, et al. 2276 (MEXU); Mpio. San Andrés Tuxtla, Laguna Escondida, 3 km NO de la Estación de Biología Tropical Los Tuxtlas. Col.: G. Ibarra 2219; LOTE 67. Estación de Biología Tropical Los Tuxtlas, G. Ibarra 2702 (MEXU). - Guatemala: Depto. Alta Verapaz, Sebol, E. Contreras 5385 (MEXU); Depto. Izabal: Cadenas/Pueto Mendez, on Rio Dulce Road, 8 km, Elías Contreras 9852 (MEXU); Livingston, Quebrada seca. M Veliz No. MV 98.6461 (MEXU). - Honduras: Depto. Gracias a Dios, proposed Reserva de la Biosféra Tawahka Asangni, middle drainage of Rio Patuca. Coord.: $15^{\circ} \mathrm{N}, 84^{\circ} \mathrm{W}$, Paul House 2713 (BM, MEXU); Depto. Colón Trujillo, Pat O'Mara's property behind Bario Central Trujillo, Janice Saunders 697 (MEXU). - Panama: Prov. Chiriqui, Haras San Miguel, just N of Concepcion, pastureland. J. P. Folsom 3940 (MO, MEXU).

\section{Ditrichanthus Borhidi, E. Martínez et Ramos, genus novum, hoc loco (sect. Palicoureeae)}

Typus generis: Palicourea seemannii Standl., Publ. Field Mus. Nat. Hist. Bot. Ser. 7: 240 (1931).

Frutex vel arbor parva 2-5 $\mathrm{m}$ alta, stipulae 6-12 $\mathrm{mm}$ longe in vaginam connatae cum lobis binis late triangularibus 4-6 mm longis, glabrae vel margine ciliatae,

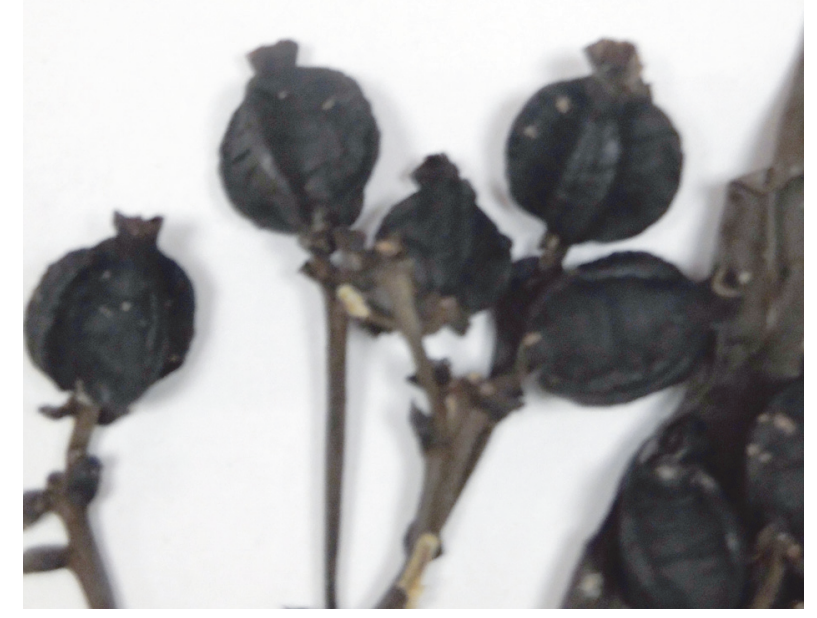

Fig. 9. Mexocarpus tetragonus, infrutescence (photo: A. Borhidi) 
persistentes et cum aetate lignescentes. Folia petiolata, lamina elliptica, oblanceolata vel obovata, chartacea subra glabra, subtus ad nervos pubescens. Inflorescentiae paniculatae, terminales, verdes, caeruleae vel violaceae, valde ramosae partiales cymosae, flores homostyli, pedicellati, bracteis triangularibus brevibus suffulti. Hypanthium obconicum, glabrum vel puberulum, calyx 5-lobulatus, lobi calycis clare evolutis, corolla 10-15 mm longa, tubus 7-12 mm longus, supra basem laeviter bullatus, extus puberulus intus villosus cum anillis duabus, unum supra base, alterum supra dimidium e fauce exertum. Stamina 5, medio glabro tubi, inter anillos duos villosos affixa, sessilia vel subsessilia, antherae lineares, supra basem dorsifixae, basi bilobulatae, filamenta brevissima, glabra e base lata abrupte angustata, stylus capitatus vel brevissime bidentatus, stigma breviter orbicularia. Fructus drupaceus manifeste 4-angulatus vel 4-costatus, obovatus 6-8 mm longus et 5-6 mm latus, pyrenae trilobatae, in sectione transversali plano-concavius triangulares, crista centrali emergente et lobis lateralibus 2 applanatis et margine leviter incrassatis margine irregulariter crenulatis.

Etymology: The generic name refers to the two hairy rings inside the corolla tube.

\section{Ditrichanthus seemannii (Standl.) Borhidi, E. Martínez et Ramos, comb. nova, hoc loco}

(Figs 10-11)

Bas.: Palicourea seemannii Standl., Publ. Field Mus. Nat. Hist. Bot. Ser. 7: 240 (1931). Holotipo: Ecuador, Seemann, sin. num. (GH). - Syn.: Palicourea copensis (Dwyer) C. M. Taylor, Fieldiana Bot., n.s. 33: 204 (1993) $\equiv$ Psychotria copensis Dwyer, Ann. Missouri Bot. Gard. 67: 365 (1980). - Palicourea mexiae Standl., Publ. Field Mus. Nat. Hist., Bot. Ser. 22: 197 (1940).

Shrubs or small treelets $2-5 \mathrm{~m}$ tall, leafy stems cylindrical, 2-5 $\mathrm{mm}$ thick, with curved or whitish hairs $0.4-0.9 \mathrm{~mm}$ long, or glabrescent. Stipules 6-12 $\mathrm{mm}$ long, united to form a broad tube to $10 \mathrm{~mm}$ long, $4-8 \mathrm{~mm}$ broad, to 14 $\mathrm{mm}$ broad below the inflorescences, bilobed with broadly triangular to ovate lobes 4-6 mm long, glabrous with a ciliolate margin, persistent and lignescent with age. Leaves with petioles 10-55 mm long, 1.3-2.3 mm thick, glabrous or sparsely puberulent beneath; leaf blades $9-30 \mathrm{~cm}, 3.5-14 \mathrm{~cm}$ broad, elliptical, oblanceolate or obovate, apex narrowly acuminate, 5-15 mm long, base cune-

Fig. 10. Ditrichanthus seemannii Borhidi, E. Martínez et Ramos. A = twig and inflorescence, $\mathrm{B}=$ flower, $\mathrm{C}=$ calyx with pistil, $\mathrm{D}=$ corolla, longitudinal section with stamens (A-D: Rueda et al. 10077, MEXU), $E$ = fruit, lateral view, $F=$ fruit, apical view, $G=$ pyrene, ventral and lateral view, $\mathrm{H}$ = cross section of fruit (E-H: Croat 49150, MEXU), 4× (drawings by M. en

$$
\text { C. Ramiro Cruz Durán) }
$$




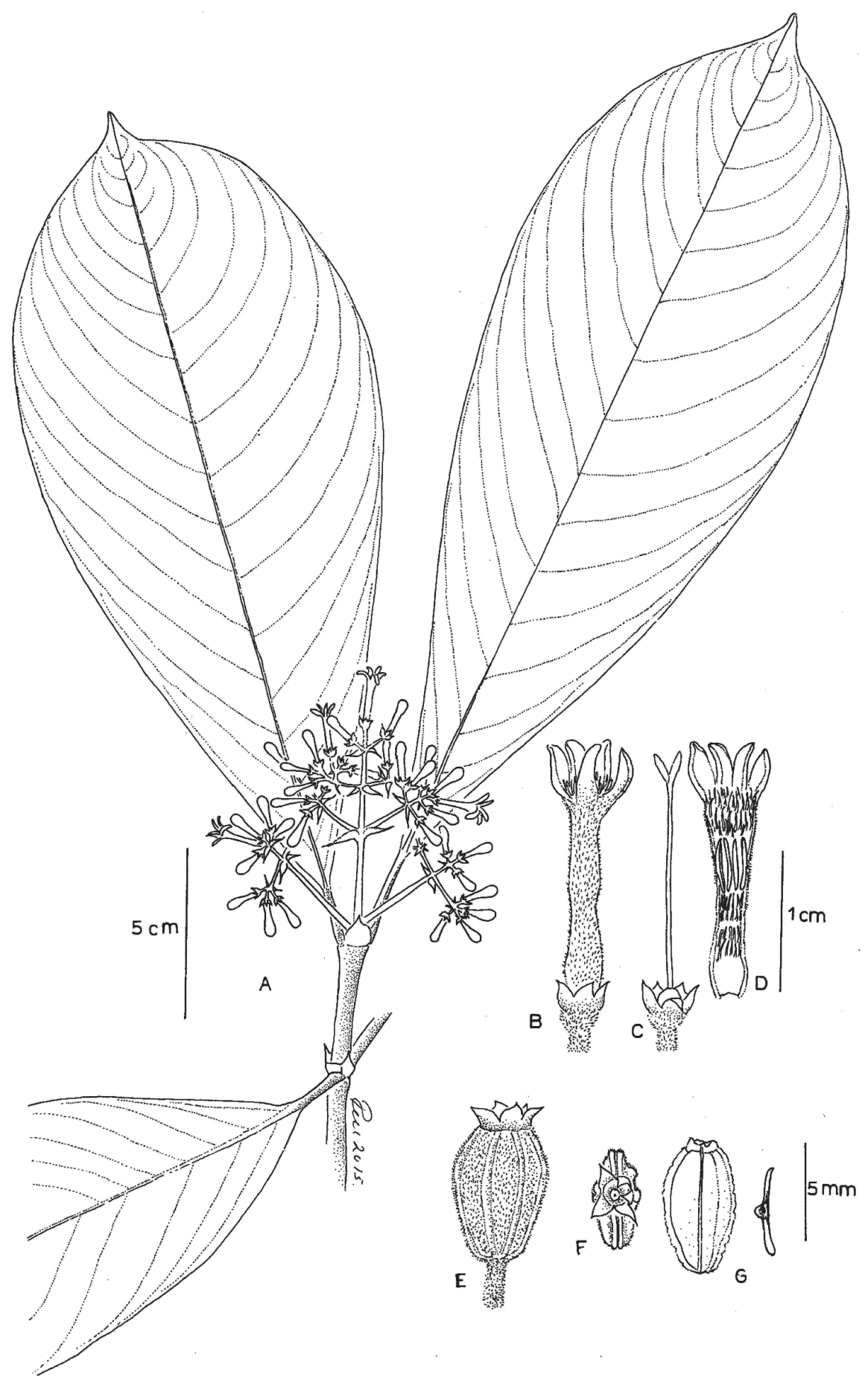


ate and slightly decurrent on petiole, drying chartaceous, dark green above, pale green beneath, glabrous above or with a few hairs along the major veins, sparsely pubescent beneath with crooked whitish hairs $0.4-1.5 \mathrm{~mm}$ long, secondary veins 9-22 pairs, arcuate-ascending distally. Inflorescences green, blue or violet, terminal or pseudoaxillary, 1 or several per node, paniculate, pilose to glabrescent, peduncles $8-45 \mathrm{~mm}$ long, pubescent or glabrous; portion floral pyramidal, richly ramified, (2-)6-14 cm long and (3-)7-22 cm broad (without inclusion of corollas), bracts narrowly triangular, 1-9 $\mathrm{mm}$ long. Flowers in distal cymose or irregular clusters, with $0.5-4.5 \mathrm{~mm}$ long pedicels. Hypanthium obconical, 1-1.2 mm long, glabrous or pubescent, calyx green or yellowish, limb 1.5-2.9 mm long, strigillous, $1 / 2$ to $2 / 3$ lobate, lobes triangular to deltoid, acute, 1-3 mm long. Corolla thick, fleshy, salverform with a slightly gibbous base, lavender, blue or purple, tube $7-15 \mathrm{~mm}$ long, sometimes white, 2-4 $\mathrm{mm}$ in diameter, puberulent or densely papillose outside, and with two strong hairy rings in the inside (Fig. 10D). The tube inside glabrous at the base, strigose-villous above the base to the middle, above the middle in the staminal range glabrous or glabrescent, above the staminal range, near the throat densely strigose-villous forming a faucial hairy ring by straight rigid exerted hairs. Corolla lobes 5, ovate or triangular, 2.5-6 mm long, slightly thickened at the apex, villous adaxially at the base, the other parts naked. Stamens 5 inserted and enclosed in the middle of the corolla tube, subsessile, filaments broad at the base, filiform at the apex, purple, glabrous, up to $1.5 \mathrm{~mm}$ long or almost absent, anthers oblong-elliptical to linear-elliptical, 3-4 $\mathrm{mm}$ long and $0.3-0.5 \mathrm{~mm}$ broad, dorsifixed in the lower tertiary, with a black point at the apex. Style filiform, 9-12 $\mathrm{mm}$ long, glabrous in the low half, densely papillose-puberulous above the middle. Stigma capitate, 1-1.2 mm long, $0.6-0.8 \mathrm{~mm}$ broad compressed ovate or obovate, densely papillose. Fruit 6-8 $\mathrm{mm}$ long, 5-6 mm broad, ellipsoidal to obovate, 4-angulate or 4-costate becoming blue or violet (Fig. 11). Pyrenes 3-lobate with an elevated central crest supplied by two longitudinal marginal membranous appendices at the apex, lateral lobes with thickened, irregularly crenulate margins; concave-triangular in section transversal. Distribution: from Nicaragua through Panama and Columbia to Ecuador.

Specimens studied: Nicaragua: Región Autónoma del Atlántico Sur, Mpio. Nueva Guinea, reserva Indio-Maiz, río Pigibaye, entre el caño bijagua y cerro Chiripa, R. M. Rueda et al. 10077 (MEXU). - Panama: Comarca de San Blas, Cerro Habú, vicinity of peak, elev. $2500 \mathrm{ft}$, cloud forest, $78^{\circ} 49^{\prime} \mathrm{W}, 9^{\circ} 23^{\prime} \mathrm{N}, 3-5 \mathrm{~m}$ tree, blue flowers, green fruit, very common. Col. K. Sytsma, T. Antonio and R. Dressler 2696, 19.12.1980 (MEXU); Province of Panamá, summit of Cerro Jefe $(1,000 \mathrm{~m}$.) down to ca $900 \mathrm{~m}$, low cloud forest (tree to $5 \mathrm{~m}$, flowers blue). Col. M. Huft \& S. Knapp 1714, 4.04.1982 (MEXU); Province of Panamá, summit of Cerro Jefe, and along road on E slope, 900-1,000 m, 79 30' W, 9 $9^{\circ} 15^{\prime} \mathrm{N}$, low cloud forest (tree $5 \mathrm{~m}$, flowers purple, tube and petal surfaces white). Col. S. Knapp \& M. Huft 


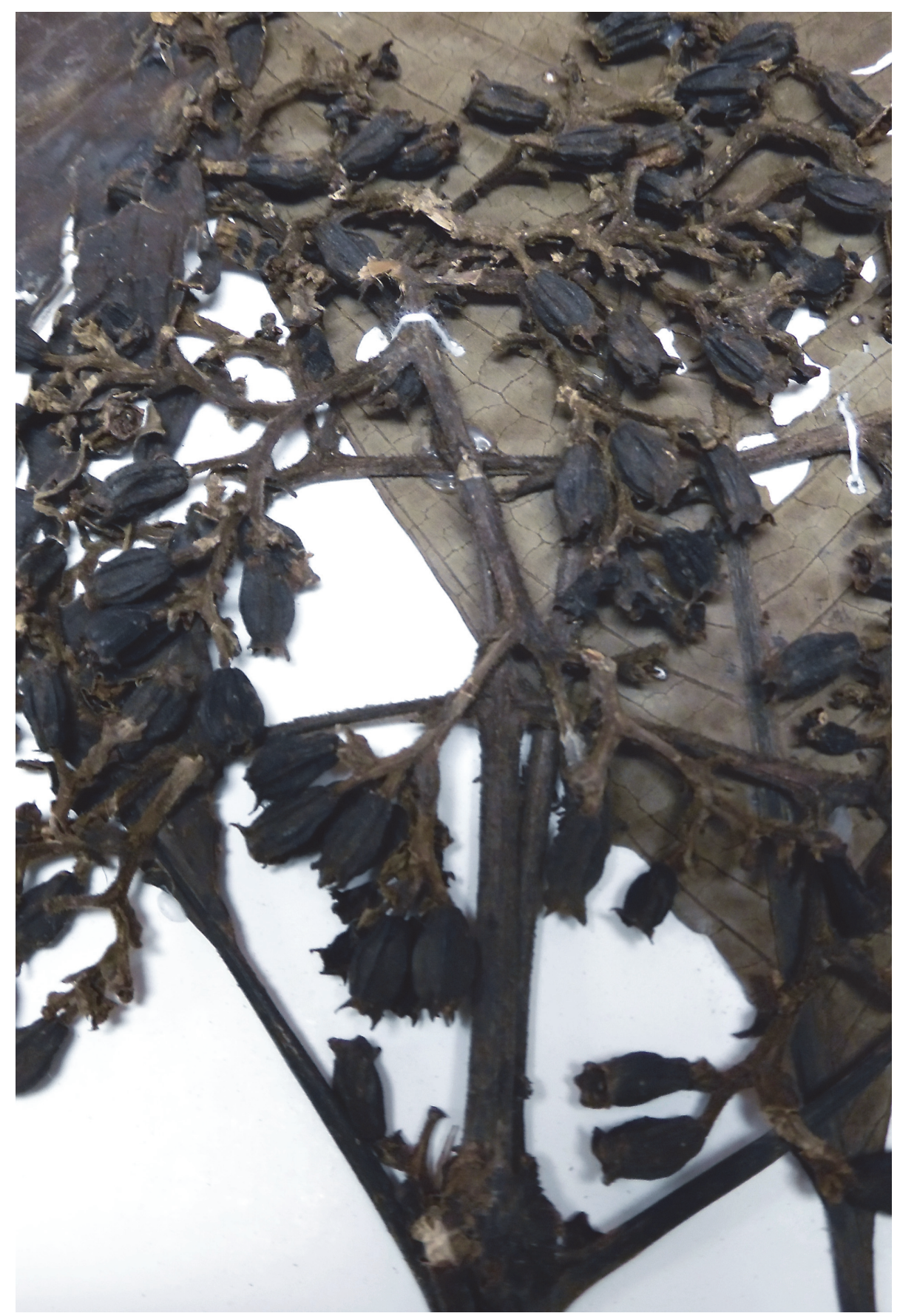

Fig. 11. Ditrichanthus seemannii, infrutescence (G. Davidse \& C. W. Hamilton 23589, MEXU) (photo: A. Borhidi) 
4571, 5.04.1982 (MEXU); Province of Cocle, area between Cano Blanco del Norte, Cano Sucio and Chorro del Rio Tife, $8^{\circ} 42^{\prime} 19^{\prime \prime}$ N, 80 36 30" W, 200-400 m elev., evergreen forest (treelet $3 \mathrm{~m}$ tall; fruit green with light green ribs). Col. G. Davidse \& C. W. Hamilton 23589, 3.02.1983 (MEXU); Province of Cocle, Alto Calvario, cloud forest above sawmill on continental divide, 5.2 miles above El Cope; elev. $930 \mathrm{~m}$ (shrub $2 \mathrm{~m}$, flowers blue, fruits green). Col.: Thomas B. Croat 49150, 6.12.1979 (MEXU); Province of Colon; South approach of Cerro Bruja from Río Escandaloso (tree $5 \mathrm{~m}$ tall, $15 \mathrm{~cm}$ dbh; flowers blue-white). Col.: B. Hammel 3115, 18.05.1978 (MEXU).

Acknowledgements - The authors express their deep thanks to Dr Gerardo Adolfo Salazar, head of the Botanical Department of the Biological Institute of the UNAM, and to Dr David Sebastian Gernandt, head of the National Herbarium of Mexico (MEXU) for the access of the studied materials of the collections and their help in creating favourable conditions for this study. The authors are very thankful to Lourdes Rico and Dr David Sebastian Gernandt for reviewing the manuscript and to M. en C. Ramiro Cruz Durán for the excellent drawings, revealing all important details.

\section{BIBLIOGRAPHY}

Borhidi, A. (2011): Transfer of the Mexican species of Psychotria subgen. Heteropsychotria to Palicourea based on morphological and molecular evidences. - Acta Bot. Hung. 53: 241-250. http://dx.doi.org/10.1556/ABot.53.2011.3-4.4

Borhidi, A. (2012): Rubiáceas de México. Segunda y ampliada edición. - Akadémiai Kiadó, Budapest, 610 pp.

Borhidi, A. and Verdcourt, B. (1990): New species of Rubiaceae-Psychotrieae from Tanzania. - Kew Bull. 54: 703-710.

Bremer, B. (2009): A review of molecular phylogenetic studies of Rubiaceae. - Ann. Mo. Bot. Gard. 96: 4-26. http://dx.doi.org/10.3417/2006197

Burger, W. C. and Taylor, C. M. (1993): Family 202. Rubiaceae. In: Burger, W. C. (ed.): Flora Costaricensis. - Fieldiana, Bot., n.s. 33: 1-333.

Dwyer, D. J. (1980a): Rubiaceae. In: R. E. Woodson, Jr., R. W. Scheryl et al. (eds): Flora of Panama. Part 1. - Ann. Mo. Bot. Gard. 67: 1-256.

Dwyer, D. J. (1980b): Rubiaceae. In: R. E. Woodson, Jr., R. W. Scheryl et al. (eds): Flora of Panama. Part IX. Family 179. Rubiaceae. Part II. - Ann. Mo. Bot. Gard. 67: 257-522.

Govaerts, R. (2014): World checklist of Rubiaceae. - Royal Botanic Gardens, Kew. http://apps. kew.org/wcsp

Nepokroeff, M. (1997): Systematics of the tropical shrub genus Psychotria L.: origins, speciation and breeding systems in Hawaii. - PhD Diss., Univ. of Wisconsin-Madison.

Nepokroeff, M., Bremer, B. and Sytsma, K. J. (1999): Reorganization of the genus Psychotria and tribe Psychotrieae (Rubiaceae) inferred from ITS and rbcL sequence data. Syst. Bot. 24(1): 5-27. http://dx.doi.org/10.2307/2419383

Petit, E. (1963): Rubiaceae africanae X. Colletoecema genre nouveau de Rubiaceae. - Bull. Rijksplantentuin (Brussel) 33: 375-380.

Petit, E. (1964): Les espèces africaines du genre Psychotria L. Rubiaceae. I. - Bull. Rijksplantentuin (Brussel) 34: 1-228. 
Piesschaert, F., Robbrecht, E. and Smets, E. (2001): Patterns in pyrenes: the systematic significance of pyrene morphology in Chassalia (Rubiaceae, Psychotrieae) and related genera. - Flora 196: 121-131.

Razafimandimbison, S. G., Taylor, C. M., Wikström, N., Pailler, T., Khodabandeh, A. and Bremer, B. (2014): Phylogeny and generic limits in the sister tribes Psychotrieae and Palicoureeae (Rubiaceae): evolution of schizocarps in Psychotria and origin of bacterial leaf-nodules of the Malgasy species. - Amer. J. Bot. 101(7): 1102-1126. http:// dx.doi.org/10.3732/ajb.1400076

Robbrecht, E. (1975): Hymenocoleus, a new genus of Psychotrieae (Rubiaceae). - Bull. Nat Plant. Belg. 45: 273-300.

Robbrecht, E. (1988): Tropical woody Rubiaceae. Characteristic features and progressions. Contributions to a new subfamilial classification. - Opera Bot. Belg. 1: 1-271.

Robbrecht, E. (1989a): Germination slits in Rubiaceae diaspores. - Progr. \& Abstr., Flora Males. Symp., Leiden, August, 1989, p. 53.

Robbrecht, E. (1989b): A remarkable new Chazaliella (African Psychotrieae), exemplifying the taxonomic value of pyrene characters in the Rubiaceae. - Bull. Mus. natn. Hist. Nat. Paris, sér. 4, 11: 341-349.

Ruiz Lopez, H. and Pavón, J. (1794): Florae Peruvianae et Chilensis Prodromus. - La Imprenta de Sancha, Madrid.

Schnell, R. (1960): Note sur le genre Cephaelis et le problème de l'évolution parallèle chez les Rubiacées. - Bull. Rijksplantentuin (Brussel) 30: 357-373.

Standley, P. C. (1918-1934): Rubiaceae. - North American Flora 32: 1-300.

Standley, P. C. (1926): Rubiaceae. In: Trees and shrubs of Mexico. - Contr. U.S. Natl Herb. 23: $1349-1394$.

Standley, P. C. (1928): New plants from Central America XI. - J. Wash. Acad. Sci. 18: 160-169.

Standley, P. C. (1930): Studies on American plants IV. - Publ. Field Mus. Nat. Hist., Bot. Ser. 8: 133-236.

Standley, P. C. (1938): Rubiaceae. In: Flora of Costa Rica. - Publ. Field Mus. Nat. Hist., Bot. Ser. 18: 1264-1380.

Standley, P. C. (1940a): Studies on American plants X. - Publ. Field Mus. Nat. Hist., Bot. Ser. 22: $101-132$.

Standley, P. C. (1940b): Studies on American plants XI. - Publ. Field Mus. Nat. Hist., Bot. Ser. 22: $180-218$.

Standley, P. C. and Steyermark, J. A. (1940a): Studies on Central American plants I. - Publ. Field Mus. Nat. Hist., Bot. Ser. 22: 219-322.

Standley, P. C. and Steyermark, J. A. (1940b): Studies on Central American plants II. - Publ. Field Mus. Nat. Hist., Bot. Ser. 22: 358-387.

Standley, P. C. and Steyermark, J. A. (1947): Studies on Central American plants III. - Publ. Field Mus. Nat. Hist., Bot. Ser. 23: 3-28.

Steyermark, J. A. (1972): Rubiaceae. In: Steyermark, J. A., Maguire, B., Wurdack, J. J. et al.: Botany of the Guyana Highland, Part IX. - Mém. New York Bot. Gard. 23: 227-832.

Steyermark, J. A. (1974): Rubiaceae. - In: Lasser, T. and Steyermark, J. A. (eds): Flora de Venezuela. Inst. Bot., Caracas, Vol. 9(1-3), 2070 pp.

Taylor, C. M. (1989): Revision of Palicourea in Mexico and Central America. - Syst. Bot. Monogr. 26: 1-102. http://dx.doi.org/10.2307/25027720

Taylor, C. M. (2004): Rubiacearum Americanarum Magna Hama Pars XVI. New species, a new subspecies, and an overlooked species of Psychotria subg. Heteropsychotria from Mexico, Central America, and western South America. - Novon 14(4): 493-508. 
Taylor, C. M. (2005): Margaritopsis (Rubiaceae, Psychotrieae) in the Neotropics. - Syst. Geogr. Pl. 75(2): 161-177. http://dx.doi.org/10.2307/3668574

Taylor, C. M. (2012a): Palicourea Aubl. - In: Davidse, G., Sousa, M., Knapp, S. and Chiang, F. (eds): Flora Mesoamericana, Vol. 4/2, pp. 163-181.

Taylor, C. M. (2012b): Psychotria L. - In: Davidse, G., Sousa, M., Knapp, S. and Chiang, F. (eds): Flora Mesoamericana, Vol. 4/2, pp. 192-241.

Taylor, C. M. and Lorence D. H. (1985): Lectotypification of Palicourea galeottiana M. Martens (Rubiaceae) and a new name for this common species. - Taxon 34: 667-669.

Taylor, C. M. and Lorence, D. H. (1992): Notes on Psychotria subgenus Heteropsychotria (Rubiaceae: Psychotrieae) in Mexico and Northern Central America. - Novon 2: 259-266.

Taylor, C. M., Lorence, D. H. and Gereau, R. E. (2010): Rubiacearum Magna Hama Pars XXV: The nocturnally flowering Psychotria domingensis-Coussarea hondensis group plus three other Mesoamerican Psychotria transfer to Palicourea. - Novon 20(4): 481-492. http://dx.doi.org/10.3417/2009124

Verdcourt, B. (1976): Rubiaceae (part 1). - In: Polhill, R. M. (ed.): Flora of Tropical East Africa. Agents for Overseas Governments and Administration, London, 414 pp. 